Enrique Samanamud Valderrama*

Recibido: 18 de noviembre de 2020

Evaluación: 24 de agosto de 2021

Aprobado: 15 de julio de 2021

Artículo de investigación

(C) 2021 Universidad Católica de Colombia.

Facultad de Ciencias

Económicas y Administrativas.

Todos los derechos reservados

* Master en Finanzas Públicas. Docente e investigador del Instituto de Investigación Científica (IDIC) de la Universidad de Lima, Perú.

(D) https://orcid.org/0000-0002-3380-1504
Revista Finanzas y Política Económica, Vol. 13, N. ․ 2, julio-diciembre, 2021, pp. 473-511 https://doi.org/10.14718/revfinanzpolitecon.v13.n2.2021.8

\section{Una revisión para el Perú de la relación entre el desempleo, el subempleo y la producción}

\section{Resumen}

Este artículo examina la relación entre el desempleo y la producción en el Perú entre 1970 y 2018 —denominada comúnmente como ley de Okun-, a partir de las dos primeras versiones planteadas originalmente por Arthur Okun. Asimismo, se plantea una nueva formulación econométrica que agrega al desempleo el subempleo como variable dependiente, buscando una aproximación a los elevados niveles de empleo precario característicos de economías en desarrollo, que sirven de vía de escape a la población y evitan mayores niveles de desempleo. Finalmente, a los modelos se incorporan variables adicionales al producto que permitan un mejor ajuste. Los resultados evidencian inferiores niveles del coeficiente en comparación con los estimados originalmente para la economía de Estados Unidos y de similares trabajos efectuados para las economías desarrolladas por diversos autores, lo que implica mayores niveles de producto para reducir el desempleo. Además, la reformulación agregada de desempleo con subempleo ayuda a explicar mejor las necesidades de crecimiento de la economía peruana.

Palabras clave: brecha de producto, desempleo, ley de Okun, Perú, subempleo.

JEL Classification: C22, E24, E26, E32, J01 


\section{A review for Peru of the relationship among unemployment, underemployment and production}

\section{Abstract}

This paper examines the relationship between unemployment and production in Peru between 1970 and 2018, commonly referred to as Okun's Law, based on the first two versions originally proposed by Arthur Okun. Likewise, a new econometric formulation is proposed, adding underemployment as a dependent variable to unemployment, seeking an approximation to the high levels of precarious employment characteristic of developing economies, which serve as an escape route for the population and avoid higher levels of unemployment. Finally, additional variables to the output are incorporated into the models to allow for a better adjustment. The results show lower levels of the coefficient, in reference to those originally estimated for the United States economy and similar studies carried out for developed economies by various authors, which implies higher levels of output to reduce unemployment. Furthermore, the aggregate reformulation of unemployment with underemployment helps to better explain the growth needs of the Peruvian economy.

Keywords: Okun's Law, unemployment, underemployment, production gap, Peru. 


\section{INTRODUCCIÓN}

En 1962 Arthur Okun se propuso explorar cuánto podría producir Estados Unidos estando en condiciones de pleno empleo, lo que se conoce como el Producto Potencial; formuló así que existe una relación bidireccional entre la tasa de desempleo y el crecimiento del Producto Nacional Bruto (PNB), y para demostrarlo planteó hasta tres modelos de estimación. A pesar de las críticas metodológicas, la formulación empírica de Okun encontró cierta regularidad y validez en la mayoría de las economías desarrolladas, y por tal razón es considerada hoy en día como una ley, además de constituirse en una de las relaciones económicas más exploradas y reformuladas junto a la curva de Phillips (aunque con mayor suerte que esta última).

Posteriormente, se han efectuado diversos estudios que proponen diferentes versiones y adaptaciones al modelo; sin embargo, algunos de los realizados en economías de países en desarrollo han encontrado que el impacto del producto sobre el desempleo, expresado a través del coeficiente de Okun, dista del nivel obtenido originalmente. La explicación de esta diferencia quizá se pueda encontrar en las características estructurales propias de sus mercados de trabajo, con un acelerado crecimiento de la oferta de mano de obra y bajos niveles de productividad, segmentación, elevada informalidad laboral ${ }^{1} \mathrm{y}$ empleos precarios, entre otros.

En este contexto, el presente artículo tiene como principal objetivo determinar el valor del coeficiente para Perú, de acuerdo con las especificaciones de los dos primeros modelos teóricos originales de Okun. Al respecto, cabe mencionar que en el 2003 se publicó una investigación que explora la relación original de Okun para Perú, la cual se revisará en la parte de literatura especializada. El otro objetivo es incorporar un nuevo planteamiento que permita obtener un coeficiente que se ajuste mejor a las características del mercado laboral peruano, y para ello se desarrolla el concepto de desempleo ampliado.

Con esta finalidad, la investigación se divide en cinco partes. En la primera se efectúa una revisión de la literatura sobre dicha relación. En la segunda se hace un análisis sobre el mercado de trabajo en el Perú. En la tercera se expone el marco conceptual. En la cuarta se propone la metodología de la investigación. En la quinta se presentan y analizan los resultados obtenidos de cada grupo de modelos empíricos. Finalmente, se cierra el artículo con las conclusiones.

1 En este caso, la definición de la informalidad es la que se utiliza desde hace algunas décadas en Perú, es decir, la que se refiere a todo tipo de empleo que no cuenta con los beneficios estipulados por ley, como los beneficios de seguridad social, vacaciones pagadas, licencia por enfermedad, etc. 


\section{REVISIÓN DE LA LITERATURA}

\section{Los modelos originales de Okun}

En su célebre trabajo seminal titulado Potential GNP: Its measurement and significance [PNB potencial: su medida y significado], Okun (1962) plantea, sobre una muestra completa de 55 observaciones entre 1947.II y 1960.IV, el primer modelo conocido como el de primeras diferencias, que relaciona en forma trimestral la variación de la tasa de desempleo $\left(\Delta D_{t}\right)$ sobre la variación porcentual del producto efectivo real $\left(\Delta Y_{t}\right)$. Su ecuación formulada fue la siguiente:

$$
\Delta D_{t}=\beta_{0}+\beta_{1} \cdot \Delta Y_{t}+\varepsilon_{t}
$$

Según esta estimación, la tasa de desempleo aumentará en 0,3\% de un trimestre al siguiente si el producto real no se reduce, ya que las ganancias seculares en la productividad y el crecimiento de la fuerza laboral elevan la tasa de desempleo. Asimismo, por cada punto porcentual adicional de variación del producto, el desempleo se reduce 0,3 puntos porcentuales. En resumen, para que el desempleo se reduzca un punto porcentual, se requiere al menos de 3,3\% de aumento en la variación del producto real.

El segundo modelo es el de un enfoque de brechas que prueba ciertas trayectorias exponenciales del producto potencial, utilizando tasas de crecimiento alternativas asumidas y niveles de referencia, como que el producto potencial debía ser igual al producto real cuando el desempleo era de $4 \%(D=4)$, considerada la tasa de desempleo natural de común aceptación durante la época de su estudio. Dicho modelamiento es el siguiente:

$$
\left(D_{t}-D_{t}^{*}\right)=\beta_{0}+\beta_{1} \cdot\left(Y_{t}-Y_{t}^{*}\right)+\varepsilon_{t}
$$

Donde $D_{t}$ es el desempleo efectivo, $D_{t}^{*}$ es el desempleo natural, $Y_{t}$ es el producto efectivo real y $Y_{t}^{*}$ es el producto potencial del periodo $t$.

En esta estimación, un incremento del desempleo del 1\% está asociado con una pérdida de producción igual al 2,8\% de la producción potencial, o un porcentaje algo mayor de la producción real cuando está por debajo del potencial. La tasa de desempleo estimada asociada con una brecha del producto de cero en este caso es del 3,72\%, no muy lejos del ideal del 4,0\%. 


\section{Estudios aplicados del coeficiente de Okun para distintos países y periodos}

Luego del estudio de Okun en 1962, innumerables investigadores probaron empíricamente dicha formulación especialmente para otras economías. Se pueden mencionar inicialmente las estimaciones de Hamada y Kurosaka (1984) para la economía japonesa de posguerra: encontraron un coeficiente sumamente grande comparado con los obtenidos para los Estados Unidos, llegando a 28,2\%, lo que genera una respuesta relativamente pequeña del empleo a la producción, esto es, de $0,035 \%$. Fortin y Bernier (1988), para la provincia de Québec en Canadá, sobre una muestra de 34 individuos representativos, obtienen en sus resultados un sesgo de sobrestimación del costo del desempleo cíclico, medido por el coeficiente de la brecha del producto, que varía entre $3 \%$ y $20 \%$.

Holloway (1989), para Estados Unidos, con datos trimestrales de 1970.1 a 1989.4, estima el coeficiente utilizando el tercer método propuesto por Okun, y encuentra que las tasas de crecimiento real del PNB que superen el 2,25\% reducirían la tasa de desempleo, o dicho de otra forma, que para reducir el desempleo en un $1 \%$, la tasa de crecimiento del producto debía ser de al menos 4,11\%.

Barreto y Howland (1993), con datos trimestrales para Estados Unidos de 1947:1 a 1960:4, demostraron que no era válido utilizar el recíproco de la pendiente para predecir el efecto de la producción, dado un nivel de desempleo, a partir de una regresión inversa del efecto de la producción sobre el desempleo. Por esta razón, señalan que las estimaciones hechas arrojan un valor del coeficiente que resulta muy alto, dado que se pretende calcularlo a partir de la inversa del coeficiente del producto, que resulta sustancialmente bajo cuando se toma la variable desempleo como dependiente, lo que resulta poco confiable. Dichos autores proponen que, si se quiere usar la ley de Okun en un modelo econométrico para pronosticar el nivel de desempleo en respuesta de cambios anticipados del nivel de PNB real, lo correcto será estimar el desempleo en función del PNB. Finalmente, recomiendan que lo más apropiado para estimar el coeficiente de Okun es que se realice un modelo de ecuaciones simultáneas.

Izyumov y Vahaly (2002), para 25 países en transición de la llamada Europa del Este, con datos anuales de 1991 al 2000, determinan que la tasa de crecimiento del producto debe ser de al menos 3,31\% para que el desempleo no se eleve, de alrededor de un $11 \%$ para reducir la tasa de desempleo en un punto porcentual, 
y que los países que iban más rápido por la senda de transición hacia una economía de mercado presentaban una relación más rápida entre el desempleo y la producción. En contraste, los rezagados en salir de la planificación centralizada parecían llevar las rigideces de su mercado laboral durante un largo periodo en el tiempo posterior a la reforma.

Knotek (2007), usando data para la economía norteamericana de frecuencia trimestral entre 1948.2 y 2007.2, concluye que la ley de Okun no ha sido una relación estable a lo largo del tiempo, sino que está conectada con las fases de la economía, lo cual apoya la hipótesis de asimetría del coeficiente. Adicionalmente, observa que la relación entre producto y desempleo en el mismo periodo se ha debilitado, mientras que se ha fortalecido la relación entre producto pasado y desempleo contemporáneo.

Loría y Ramos (2007), con datos anuales para México entre 1970 y 2004, indican que el coeficiente de Okun se encuentra en el intervalo de 2,08 a 2,5, lo cual es un resultado congruente con una economía que sufrió de alto desempleo, fue intensiva en fuerza de trabajo y reportó baja productividad. Anderton et al. (2014), con datos trimestrales de 17 países de la zona euro durante el periodo 1996.1-2013.4, sugieren que el desempleo en la zona es particularmente sensible a las variaciones de los componentes de consumo del PNB, aunque las variables relacionadas con el exterior (exportaciones e importaciones) tienen un impacto mucho menor sobre la evolución del desempleo.

Valadkhani (2015), empleando datos de frecuencia trimestral para Australia de 1980.3 a 2014.1, concluye que el coeficiente de Okun está entre 2,26 y 2,38 en valor absoluto, y que existe asimetría en la muestra analizada, donde la relación entre producto y desempleo es mayor en las recesiones. Por su parte, Jiménez y Ochoa (2017), para 12 países de América Latina, con data anual durante el periodo 1991-2016, concluyen que para el conjunto de países existe una relación inversa entre crecimiento (PIB) y desempleo. Además, que las fluctuaciones cíclicas tienen un efecto permanente sobre el nivel de la tasa de desempleo; es decir, que existe rigidez en el mercado de trabajo, con lo cual se comprueba la hipótesis de histéresis. ${ }^{2}$ Bande y Martín-Román (2018), utilizando datos para 17 regiones en España de

2 La histéresis es un concepto que, empleado en economía, suele hacer referencia — sobre todo en el mercado laboral - a la persistencia del desempleo en el tiempo. Que la tasa de desempleo aumente en medio de una recesión es algo normal, por los efectos derivados sobre el consumo, inversión, etc., de un ciclo recesivo; pero si luego de unos años de haber finalizado la recesión, el mercado laboral sigue mostrando altos niveles de desempleo, se dice que se debe a la aparición del fenómeno de histéresis, es decir, que las fluctuaciones cíclicas pueden tener un efecto permanente sobre el nivel de la tasa de desempleo, entre otras razones, por la pérdida de habilidades de la fuerza laboral requeridas por la demanda de empleo. 
1980 a 2015, encuentran asimetría en la muestra, lo cual varía significativamente el tamaño del coeficiente dependiendo de si se está en la fase expansiva o recesiva.

Mención especial merece para el caso del Perú el trabajo de Garavito (2003), quien realiza dos estimaciones de la ley de Okun, con datos anuales entre $1970 \mathrm{y}$ 2000, para el caso del desempleo a nivel de Lima Metropolitana. La primera estimación corresponde al modelo de elasticidad del empleo, a través de un modelo de cointegración con método de corrección del error (MCE); se encuentra así una relación positiva de largo plazo entre la tasa de empleo y el PIB de Lima Metropolitana, donde por cada S/1000 nuevos soles de 1979 de elevación del producto, la tasa de empleo se elevará en 0,000288 unidades o 0,0288 puntos porcentuales, con una velocidad de ajuste de 0,222824 . La segunda estimación se corresponde con el segundo modelo de Okun de brechas, determinando un coeficiente de Okun de -0,079, que considera un nivel bastante bajo y solamente comparable a los coeficientes de Japón, Bolivia y Argentina; esto se explica a partir del comportamiento procíclico de la fuerza laboral y al hecho de que, al perder su empleo, los trabajadores se retiran del mercado laboral.

En resumen, la exploración y el análisis de las numerosas investigaciones realizadas previamente nos permiten identificar algunos elementos importantes sobre el estado del arte en esta materia:

a. Algunos mecanismos institucionales que causan mayor o menor rigidez en los mercados de trabajo, además del producto, pueden tener impacto sobre la tasa de desempleo.

b. En los países con un alto nivel de economía informal, el indicador de Okun siempre resulta más pequeño, a pesar de que ello depende del carácter procíclico o anticíclico del empleo informal y de su interacción con el desempleo en el mercado formal.

c. El coeficiente de Okun tiene mayor valor durante la fase contractiva del ciclo económico, respecto de la fase expansiva, lo cual indica un comportamiento asimétrico (no lineal).

d. El desempleo puede presentar un fenómeno de histéresis, lo cual contradice la formulación teórica clásica que define la existencia de una tasa natural de desempleo, es decir, únicamente de desempleo voluntario, pero sí está de acuerdo con la más moderna tesis de la NAIRU. ${ }^{3}$

3 Tasa de desempleo no aceleradora de la inflación. 


\section{ESTRUCTURA DEL MERCADO DE TRABAJO EN EL PERÚ}

Un primer elemento estructural del mercado de trabajo en el Perú es la transformación en la composición demográfica, marcada por el final del bono demográfico, es decir, de mayor crecimiento de la población en edad activa en relación con la población infantil-juvenil y de adultos mayores, y que representa siempre una situación favorable para la economía de un país. El final de esta etapa tendrá un impacto importante en el mercado de trabajo a largo plazo, ya que ampliará la brecha entre la demanda y la oferta en el mercado laboral. De hecho, la relación de dependencia que mide la carga demográfica relativa de los niños y de las personas de mayor edad, sobre la población en edades activas, muestra su nivel mínimo $(0,502)$ hacia el año 2020.

Una segunda característica estructural es la segmentación del mercado de trabajo, con un sector económico moderno capitalista donde prima una relación laboral dependiente y otro tradicional de trabajadores que se autoemplean. Cada uno de estos sectores de la economía tiene características muy diferentes: en el primero existe una mayor productividad y salarios marcadamente superiores; y en el segundo, una insuficiente utilización de capital, de desarrollo tecnológico y altos niveles de informalidad laboral y empleos precarios. Finalmente, una desarticulación económica y escasa movilidad laboral entre ellos.

La tercera característica es el bajo nivel de productividad de la fuerza laboral, comparado tanto con otros países como entre sectores dentro del mismo país. En un estudio de Távara et al. (2014) se señala que, luego de una etapa de decrecimiento que se inició en 1976, la productividad ha venido creciendo sostenidamente desde inicios de la década de 1990. Esto es lo que se desprende también del estudio de Céspedes y Ramírez (2016), que estiman la productividad total de los factores y determinan que, al menos entre el 2003 y 2012, la participación del trabajo ha mostrado una tendencia creciente entre el inicio y fin de la década estudiada. Sin embargo, de acuerdo con estadísticas obtenidas de los microdatos de la Organización Internacional del Trabajo (ILOSTAT), se encuentra que la producción por trabajador del Perú es de \$22.868 para el 2018, lo cual está por debajo del promedio de países de América Latina y el Caribe, de $\$ 32.696$.

Una cuarta característica es la presencia de una elevada heterogeneidad a nivel de los estratos empresariales del país, que se hace visible con la existencia abundante de micro y pequeñas empresas. Su importancia radica en ser una gran fuente generadora de empleos, es el sector donde se concentra el mayor problema 
de baja productividad y alta tasa de informalidad. En las microempresas, cerca del $30 \%$ poseen un solo trabajador, y el resto contrata entre 2 y 10 trabajadores, que las estadísticas sugieren que en muchos casos suelen ser familiares o desempleados que aceptan un empleo u oficio no acorde con su nivel de instrucción.

Finalmente, la quinta característica es su alto nivel de informalidad laboral y precariedad del empleo, lo cual implica relaciones de trabajo que no cumplen con la regulación vigente de forma parcial o total, tales como el pago del salario mínimo, los pagos a la seguridad social en salud, al sistema de pensiones, vacaciones, protección contra el desempleo, etc. La informalidad laboral se puede situar en unidades de producción formal e informal, es decir, no registradas, alcanzándose, según el INEI (2017), un nivel de 72\% en el 2016, que incluye un 55\% de empleo informal en el sector informal y un $17 \%$ de empleo informal fuera del sector informal.

Por su parte, un informe de Belapatiño et al. (2017) estima la tasa de informalidad consistente con las características particulares de Perú en referencia al marco institucional, cumplimiento de las leyes y otras características. Los autores sugieren que un país como el Perú debería tener una informalidad laboral en torno al 40\%; "por lo tanto, no solo la informalidad laboral es alta en términos absolutos, sino que parece ser también mayor que la de otros países con características similares" (p. 3).

En el Perú, tal como se muestra en la figura 1, la Población en Edad de Trabajar (PET) se subdivide en PEA, que es la Fuerza de Trabajo o Población Económicamente Activa, y la Población Económicamente Inactiva (PEI). Esta última incluye, además de los inactivos plenos, los llamados desalentados, que son aquellos que, por falta de motivación, oportunidades, o por requisitos que impone el mercado y que no creen poder cumplir, han dejado de buscar activamente empleo, porque de cierta forma han perdido la esperanza de poder encontrar uno, pero que si se les ofreciera la posibilidad de tenerlo aceptarían; estos son considerados como parte de un desempleo oculto. Según Chacaltana (2000), basado en información del Ministerio de Trabajo y Promoción del Empleo (MTPE), este desempleo oculto alcanzaría el $4,8 \%$ de la PET, muy cerca a la tasa de desempleo abierto de la década de los 90 y superior a las de la última década.

La PET ha venido creciendo sostenidamente desde la segunda década de 1960 respecto del total de población, y lo mismo la tasa de actividad (PEA/PET), que en el 2018 fue del 72,3\%. La PEA o fuerza laboral (L) comprende a la población ocupada o empleada (E) y a los que se encuentran desocupados o desempleados (D), pero buscando activamente un trabajo. 
La tasa de desempleo en el Perú para el 2018, como se observa en la figura 2, fue de 3,9\% sobre la PEA, por género más alta en las mujeres, con 4,1\%, y en los jóvenes de 14 a 24 años, con 11,1\%. Por nivel educativo, el desempleo alcanzó un $5,6 \%$ entre los que tienen educación universitaria, seguido de los que alcanzaron educación superior no universitaria y secundaria, ambos con un 4,6\%. Esta tasa de desempleo en el 2018, según el Fondo Monetario Internacional, resultó de las más bajas de América Latina, por encima de Colombia, con una tasa de 9,13\%; Brasil, con $12,26 \%$; Chile, con 6,94\%; Paraguay, con 6,23\%, y Uruguay, con 8,38\%.

Figura 1. Perú: clasificación del mercado laboral en el 2018

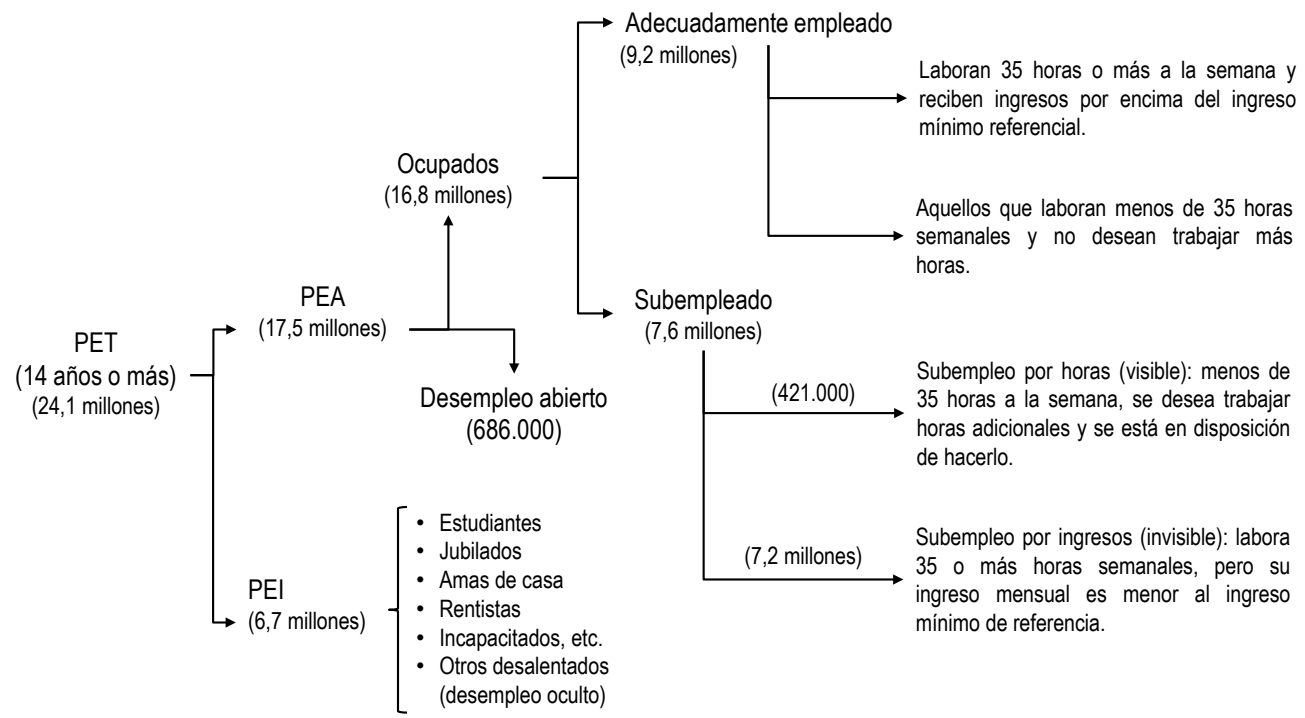

Fuente: elaboración del autor a partir de información del INEI.

La tasa de empleo (E/PET) en el 2018 fue de 69,7\%, superior a la de América Latina y el Caribe, de 57,3\% (Organización Internacional del Trabajo [OIT], 2018). La población empleada se divide entre aquellos que se encuentran adecuadamente empleados (A) y los subempleados (S). Los adecuadamente empleados significaron en el 2018 el 54,7\% del total de ocupados, y en términos de género, la población masculina significó el $60,5 \%$, lo que muestra una preferencia del mercado por contratar formalmente a hombres antes que a mujeres. La población subempleada corresponde al 43,6\% de la PEA. La proporción de la población empleada masculina 
que en el 2007 se encontraba subempleada era de 35,6\%, en tanto la femenina alcanzaba el $56,1 \%$.

Figura 2. Perú: subempleo y desempleo entre 1970 y 2018 1/

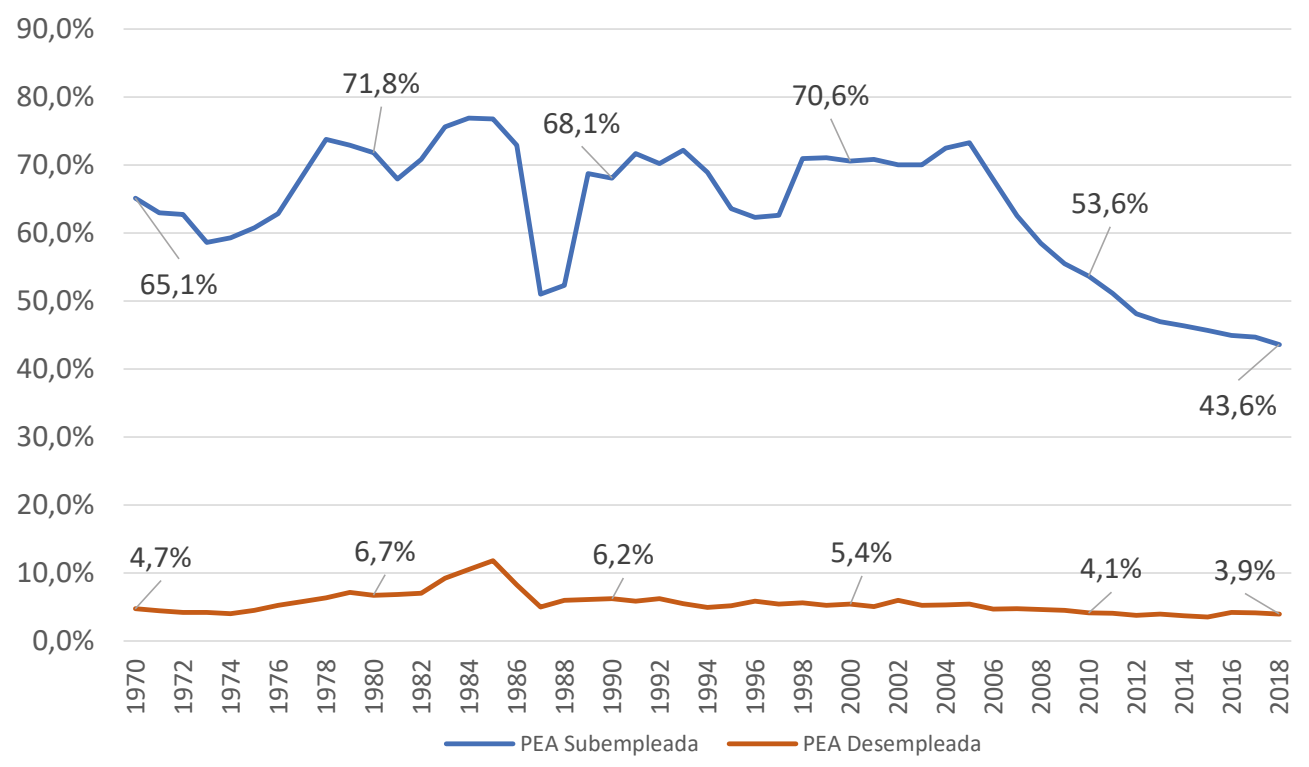

1/: Como proporción de la PEA

Fuente: elaboración del autor a partir de información del INEI y MTPE.

A su vez, podemos distinguir dos tipos de subempleo: el llamado subempleo por horas o visible, que refleja una insuficiencia en el volumen de empleo (jornada parcial de trabajo), y el subempleo por ingresos o invisible, caracterizado por los bajos ingresos que perciben los trabajadores. En el Perú, para el 2018 la mayoría de los trabajadores subempleados son del tipo invisible, que asciende a 7,2 millones y corresponde al $41,1 \%$ de la PEA.

\section{MARCO CONCEPTUAL}

Como se vio en el apartado anterior, en el Perú el desempleo es inferior al de otros países de la región, porque encubre un desempleo oculto entre los inactivos y un empleo informal y de mala calidad, aproximado por la medición del subempleo; ambos amortiguan la relación entre la tasa de desempleo abierto sobre la base 
del producto. Tratar de incorporar este elemento no resulta sencillo; sin embargo, podemos tratar de agregar el subempleo al desempleo abierto como una forma de incorporarlo en el análisis. La figura 3 muestra los flujos de empleabilidad del mercado laboral en cada una de las etapas del ciclo económico. En ella se observa la existencia de un desempleo encubierto dentro del componente de la $\mathrm{PEI}$ - que algunos denominan como los "desalentados"-, que principalmente se activa cuando la economía crece; algo a lo que podríamos denominar un "efecto de absorción".

Figura 3. Flujos de empleabilidad en el mercado de trabajo
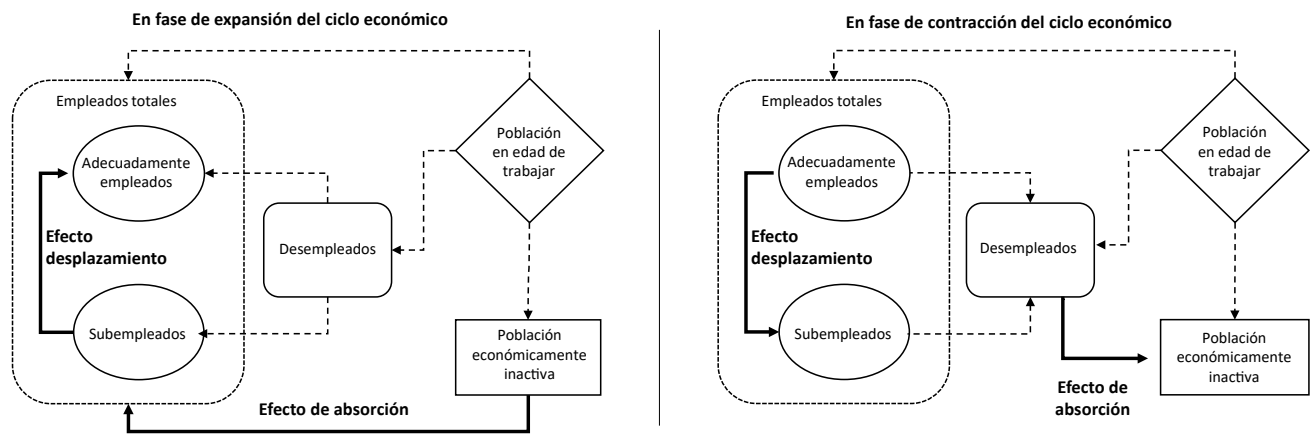

Fuente: elaboración del autor.

Asimismo, ante variaciones en la tasa de crecimiento de la economía dentro de la fuerza laboral (L), existe un trade-off entre los que trabajan en condiciones de formalidad total y los empleos informales, que de forma simplificada podemos relacionar mayormente con los adecuadamente empleados y el subempleo, respectivamente; ${ }^{4}$ es decir, cuando estamos en la fase expansiva del ciclo, ocurre no solo una reducción del desempleo, sino también una reducción del subempleo que se desplaza hacia los adecuadamente empleados, y que ocurre lo inverso cuando estamos en la fase contractiva, a lo que llamaremos como "efecto desplazamiento".

4 Como se ha mencionado, el empleo informal se encuentra presente dentro de los adecuadamente empleados y dentro de los subempleados; sin embargo, ante la inexistencia de series estadísticas suficientes sobre empleo informal, asumimos que los adecuadamente empleados son empleos parcialmente formales y que los subempleados son todos empleos informales. El estadístico de correlación de Pearson entre subempleo e informalidad laboral para el periodo de 2004-2017 —en que existe información — es de 95,5\% y la regresión entre ambas variables presenta un R2 ajustado del 92\%. Para una demostración empírica de dicha relación, ver Bardales (2011). 


\section{EI desempleo ampliado}

Para incorporar los efectos mencionados y sustentar la utilización de la variable dependiente denominada "desempleo ampliado", partimos de las siguientes identidades que surgen de la contabilidad del mercado laboral peruano. La PET (N) aumenta a medida que crece la población y se compone de la PEA o fuerza laboral (L) y de la PEI (I):

$$
N=L+I \rightarrow L=N-I
$$

La fuerza laboral, a su vez, puede estar empleada (E) o desempleada (D):

$$
L=E+D \rightarrow D=L-E
$$

Finalmente, de la población empleada, una parte está adecuadamente empleada (A) y otra subempleada (S); por tanto, se tiene:

$$
E=A+S \rightarrow S=E-A
$$

Combinando las identidades (2) y (3), resulta:

$$
D+S=(L-E)+(E-A)=L-A
$$

Dividimos entre la fuerza laboral o PEA (L) y reemplazamos por la identidad (1):

$$
\frac{D+S}{L}=1-\frac{A}{L} \rightarrow \mathrm{da}=1-\frac{A}{N-I}
$$

Esta última identidad, que denominamos "desempleo ampliado con subempleo" (DA), permite apreciar el impacto que tiene sobre el desempleo la variación del segmento de los llamados "inactivos", efecto que hemos denominado "de absorción". El ingreso de inactivos está formado por los que entran por primera vez al mercado laboral (estudiantes, por ejemplo), y en otros casos, los que se reincorporan ya sean del grupo de desalentados o aquellos que, por razones de enfermedad, maternidad $\mathrm{u}$ otra, dejaron el mercado laboral por un mediano o largo plazo, el suficiente para que las estadísticas lo recojan como inactivo, pero que, en las épocas de expansión del ciclo económico, pueden incorporarse nuevamente al mercado laboral.

Algunos ingresarán o reingresarán al mercado laboral como subempleados en trabajos eventuales por horas del día y otros como adecuadamente empleados (amas de casa que regresan una vez los hijos crecen, por ejemplo). Por esta razón, que tiene que ver con la percepción de cada uno sobre si vale considerar lo que realiza como un trabajo al momento de contestar las encuestas, el registro de este grupo 
solo se logra de forma parcial. Además, también captura a través del subempleo el traslado bidireccional que se presenta del subempleo (S) al empleo adecuado (A) en ciclos de expansión y en sentido contrario en ciclos de contracción, que hemos denominado "efecto desplazamiento".

Es necesario señalar que existen otros elementos asociados con la variación y magnitud de la tasa de desempleo y de la tasa de subempleo, como los relacionados con el nivel de actividad económica (productividad de la mano de obra, cambio tecnológico, uso del capital, etc.), o relacionados con la composición y distribución de la población (la conformación de los grupos etarios a través del tiempo, la mayor participación de las mujeres en el mundo laboral, etc.), y finalmente, factores ligados a elementos institucionales, como el control del cumplimiento de la regulación, el costo de formalizarse, etc., que afectan la proporción de empleo adecuado.

\section{Otras variables que explican al desempleo}

A efectos de sustentar la incorporación de algunas variables en el modelo empírico, adicionales a las del desempleo y el producto, podemos recurrir al proceso de ajuste del equilibrio económico, y revisar los elementos centrales que afectan la oferta y demanda agregada a corto y mediano plazo, y a partir de allí, agregarlos en la especificación de la relación de Okun.

Para ello, por el lado de la oferta agregada, nos tenemos que apartar de las formulaciones clásicas y más bien nos acercamos a las formulaciones neokeynesianas con imperfecciones del mercado de trabajo, que explican la rigidez de los salarios reales para acercarse a su nivel de equilibrio y, por ende, de la persistencia de mayores tasas de desempleo. En esta línea de pensamiento, existen variadas teorías que explican los desequilibrios en el mercado de trabajo, entre las que podemos mencionar la de los contratos implícitos (Implicit Contract Model), la de salarios de eficiencia (Efficiency Wage Theory) y la de los mercados segmentados (Labor Market Segmentation $)^{5}$

Adoptando el procedimiento seguido por Blanchard et al. (2012, pp. 149250), para especificar la oferta agregada, partimos de la ecuación de salarios reales

$5 \quad$ En la misma línea de mercados segmentados está el modelo insider-outsider, que separa a los insertados en el mercado de aquellos que están fuera y buscan insertarse. Los primeros tienen poder de mercado y, por lo tanto, la capacidad de determinar el salario por encima del equilibrio. 
desde el lado de la oferta de trabajo, donde los individuos la determinan a partir de una relación positiva con las expectativas de precios (Pe) y de otros factores, entre los cuales se cuenta la relación negativa con la tasa de desempleo (d) mediada por un coeficiente $(\alpha)$, que mide la fuerza del efecto que produce el desempleo en los salarios; es decir, mientras mayor sea el desempleo, menor será el poder de negociación, porque aumenta el costo de oportunidad de quedarse sin trabajo. También debido a la relación positiva con un factor de rigidez laboral $(\delta)$, que puede deberse a múltiples factores como la existencia de salarios mínimos, el poder de los sindicatos, la abundante regulación, las prestaciones de desempleo, etc., de acuerdo con el marco de relaciones laborales de cada país. Podemos resumir la oferta laboral a través de la siguiente expresión:

$$
w=\frac{P^{e}}{P} \cdot e^{-(\alpha d-\delta)}
$$

Por su parte, relacionada con la demanda de trabajo, podemos representar una ecuación de precios que depende positivamente del costo marginal y del grado de competencia imperfecta en el mercado de bienes, que se representa por el margen de contribución $(z)$. Podemos resumir la demanda laboral a través de la siguiente expresión:

$$
P=(1+z) \cdot C M g
$$

Si asumimos por simplificación que a corto y mediano plazo el factor capital (K) es constante, tenemos que el costo marginal es igual al salario nominal sobre la productividad marginal del empleo (E):

$$
C T=W E+r \bar{K} \rightarrow C M g=W \cdot \frac{\partial E}{\partial Y} \rightarrow C M g=\frac{W}{Y^{\prime}(E)}
$$

Si reemplazamos la expresión (6) en la (5) y asumimos, por simplificación, una función de producción lineal de rendimientos constantes a escala en el empleo, y de la productividad total de factores productivos (A) como la tecnología, la calidad de la mano de obra, etc., cuyo valor se encuentra entre 0 y 1 , del tipo siguiente, entonces obtenemos:

$$
Y=A E, \quad 0<A<1 \rightarrow Y^{\prime}(E)=A
$$

Tenemos entonces que el salario real es:

$$
P=(1+z) \frac{W}{A} \rightarrow w=\frac{A}{(1+z)}
$$


De aquí, podemos obtener la expresión de la oferta agregada; ${ }^{6}$ sin embargo, para alcanzar nuestra finalidad, igualamos las ecuaciones de salario (4) y de precios (7), dinamizamos la expresión aplicando logaritmos, restando a ambos lados $\log \left(P_{t-1}\right)$, y realizamos aproximaciones, ${ }^{7}$ para obtener la siguiente expresión de la curva de Phillips:

$$
\pi_{t}=\pi_{t}^{e}+\left[\delta+z-\log (A)-\alpha d_{t}\right]
$$

De donde despejamos la tasa de desempleo:

$$
d_{t}=\frac{1}{\alpha}\left[\delta+z-\log (A)-\left(\pi_{t}-\pi_{t}^{e}\right)\right]
$$

La expresión (8) indica que el desempleo guarda una relación positiva con la rigidez laboral $\delta$ y el grado de competencia imperfecta $(z)$; y negativa con la productividad total de los factores $(A)$ y la discrepancia entre la inflación y la inflación esperada $\left(\pi_{t}-\pi_{t}^{e}\right)^{8}$

Por su parte, la demanda agregada real dinámica puede ser expresada como:

$$
Y=\beta_{f} \cdot G+\beta_{m} \cdot \mathrm{m}
$$

Donde $G$ es la demanda autónoma, compuesta del consumo e inversión privada, el gasto público, y en una economía abierta, las exportaciones netas. Asimismo, $m=M / P$ representa la oferta monetaria real. El parámetro $\beta_{f}$ representa el multiplicador de la política fiscal, y el parámetro $\beta_{m}$ representa el multiplicador de la política monetaria; que en general son una función de la propensión marginal al consumo, de la tasa impositiva uniforme, de la sensibilidad de la demanda de saldos reales de dinero que el público desea mantener por motivos transaccionales y de especulación, y de la sensibilidad de la inversión a la tasa de interés.

Si consideramos la expresión del enfoque de brechas de Okun, resulta:

$$
\left(d_{t}-d_{t}^{*}\right)=\emptyset\left(Y_{t}-Y_{t}^{*}\right)
$$

6 Para apreciar mejor que se trata de la función de oferta agregada, podemos igualar las ecuaciones de salario y de precios, y sustituir la productividad total $(A)$ por la función de producción por trabajador $y=Y / E$. Se muestra la relación positiva entre producción y precios a corto plazo $P_{t}=P_{t}^{e} \cdot e^{-\left(\alpha d_{t}-\delta\right)} \bullet(1+z) \cdot y$, pero completamente vertical en el equilibrio estacionario de mediano y largo plazo, donde $P_{t}=P_{t}^{e}$.

$7 \quad$ Si $\log P_{t}-\log P_{t-1}=\log \left(P_{t} / P_{t-1}\right)=\log \left(1+P_{t}-P_{t-1} / P_{t-1}\right)=\log (1+\pi)$ y lo mismo sucede con los precios esperados. Además, si la tasa de inflación es suficientemente pequeña, entonces $\log \left(1+\pi_{t}\right) \approx \pi_{t}$.

8 Como se explica más adelante, si la inflación es persistente, la discrepancia inflacionaria es nula y su efecto sobre el desempleo se vuelve positivo. 
Y reemplazamos (8) y (9) en dicha expresión (10), obtenemos lo siguiente:

$$
\left\{\frac{1}{\alpha}\left[\delta+z-\log (A)-\left(\pi_{t}-\pi_{t}^{e}\right)\right]-d_{t}^{*}\right\}=\varnothing\left\{\omega\left(G_{t}+m_{t}\right)-Y_{t}^{*}\right\}
$$

Con lo que la empírica relación de Okun (11) desagregada por elementos subyacentes podría analizarse a partir de una expresión como $d=f(Y, X)$, donde el desempleo $d$ sea explicado por el producto $Y$ y un set de otras variables $X$. Dichas variables pueden ser perturbaciones que ejercen presión y desequilibran el mercado del factor trabajo y las perturbaciones temporales entre la oferta y la demanda agregada, dependiente a su vez de componentes que afectan el sector real y el sector monetario a corto plazo.

Dicho de otro modo, que las relaciones originales de Okun podrían extenderse para incluir otros elementos además del producto, que permitan un mejor ajuste y expliquen las desviaciones del desempleo respecto de su tasa natural. Por ejemplo, un indicador de la mayor rigidez en el mercado de trabajo (sobrecosto laboral que afecta la contratación), que como proxi podrían ser las horas-hombre perdidas en huelgas, cuyo signo se esperaría fuese positivo, o un indicador de la concentración de mercados, que reduce la producción y la demanda de empleo y, por ende, se esperaría que su impacto sobre el desempleo también tuviera un signo positivo.

De la misma forma, indicadores de inmigración aumentan la PEA y, por tanto, el desempleo al no poder ser absorbidas por el mercado de trabajo. La mayor productividad laboral y formación bruta de capital, cuyo impacto sobre el desempleo se esperaría fuesen negativos al afectar favorablemente el producto potencial.

Sin embargo, perturbaciones como las generadas por la política fiscal y monetaria no se espera que sean significativas, porque sólo tienen un efecto temporal de corto plazo sobre el producto, asumiendo que los agentes tienen expectativas adaptativas, o que las políticas son no anticipadas y/o no creíbles; pero cuyo efecto será nulo a mediano y largo plazo puesto que las expectativas son siempre racionales, con lo cual el producto retorna a su equilibrio de estado estacionario y con un mayor nivel inflacionario, como se describe en cualquier libro de texto de macroeconomía.

En el mismo sentido, la relación negativa que el desempleo guarda con la discrepancia entre la inflación y la inflación esperada depende del enfoque y la temporalidad. Es decir, si el incremento de precios es pequeño, no anticipado por los agentes o se entiende temporal, el efecto es el señalado, donde la inflación efectiva puede reducir el desempleo al reducirse los salarios reales; sin embargo, 
si los agentes racionales se anticipan, la discrepancia desaparece y el impacto es nulo. Mas aún, si el aumento de precios es elevado y persistente, como la que tuvo el país hacia finales de los setenta y hasta mediados de los noventa, el impacto será positivo, porque los agentes generan sobreexpectativas de aumento, lo que lo convierte en un espiral inflacionario real (profecía autocumplida). Por esta razón, se espera que el valor de la inflación sea pequeño pero positivo.

La relación de Okun resulta muy popular en el análisis macroeconómico, porque ha sido suficientemente fiable y estable durante décadas, pero también porque sintetiza de una forma sencilla una identidad que en el fondo es bastante compleja y guarda muchas otras relaciones subyacentes del funcionamiento económico.

Algunos autores como Hamada y Kurosaka (1984, p. 82), Gordon (1984, p. 545), Prachowny (1993, p. 333) y Cazes, Verick y Hussami (2013) probaron la relación de Okun a partir de un modelo de componentes de producción, en el que especificaron diversas variables adicionales, además del vínculo entre empleo y producción, como la tasa de utilización de capital, el total de horas por trabajador, la participación de la fuerza de trabajo, la productividad, el promedio de horas y la población.

Anderton et al. (2014., 2014) y Unevska y Petrovska (2019) presentan modelos econométricos alternativos para un modelo de "Okun desagregado", según el cual los movimientos en las tasas de desempleo se estiman sobre la base de cambios diferenciales en los componentes de gasto del PIB (consumo, inversión, gasto público, exportaciones e importaciones), a partir del modelo de diferencias y de brechas.

Knoester (1986) introduce la tasa de crecimiento de la fuerza laboral, y determinantes estructurales como la tasa de crecimiento del salario real, la ratio de inversión sobre el producto, y de empleo público, justificado para cuantificar la desviación entre la oferta y demanda agregadas. De forma similar, Valadkhani (2015), tal como realizaron Daly y Hobijn (2010), capturan los efectos del aumento de productividad del trabajo y salarios reales en el desempleo para evitar la obtención de estimaciones mal especificadas para el coeficiente de Okun y que permitan realizar controles de sensibilidad y darle solidez al coeficiente estimado.

Céspedes (2003, p. 202), en un trabajo que verifica la relación de Phillips, considera que la tasa de desempleo está influenciada por factores cíclicos y por factores estructurales. En el primer caso, los desequilibrios se relacionan con la 
evolución coyuntural de la actividad económica y, por lo tanto, estarían relacionadas con el ciclo económico y con la demanda de trabajo, mientras que en el segundo provienen principalmente por el lado de la oferta de trabajo. Entre los factores cíclicos considerados, se señala la tasa de inflación y el nivel de actividad; y entre los factores estructurales se identifica la tasa de participación laboral, el crecimiento demográfico, la migración rural-urbana, etc. Los resultados muestran que existe una débil relación entre la tasa de inflación y la tasa de desempleo.

Chávez (2010) desarrolla un modelo de frecuencia trimestral entre 2001 y 2009 sobre la inflación y crecimiento económico como determinantes del desempleo en Colombia, suponiendo que los agentes económicos tienen expectativas adaptativas. Almonte y Carbajal (2011) estiman la relación estructural entre el crecimiento económico en el Estado de México sobre la base del desempleo e incorpora el grado de apertura comercial, derivando a partir de dicho modelo el coeficiente de Okun, luego de verificar que existe causalidad bidireccional entre la tasa de desempleo y el crecimiento del producto.

Ball, Leigh y Loungani (2012), buscado los determinantes subyacentes del coeficiente de Okun, efectúan regresiones del coeficiente por países, sobre un índice de legislación de protección del empleo (EPL), la proporción del desempleo juvenil y la proporción del desempleo de larga duración. De igual forma, Roman y Porras (2012) regresionan las diferencias del coeficiente de Okun de varias comunidades autónomas de España con variables adicionales como el porcentaje de trabajadores por cuenta propia sobre el total de ocupados, la tasa de crecimiento media de la productividad del trabajo por ocupado en tres sectores distintos, y el porcentaje de trabajadores asalariados con contrato temporal sobre el total de asalariados.

Páez (2013) desarrolla un modelo para Latinoamérica, donde incorpora el costo de uso del capital y el salario y demuestra que inciden de manera positiva y significativa sobre la tasa de desempleo, dando cuenta de las rigideces que exhiben los salarios sobre el desempleo y, por otro lado, del efecto del encarecimiento del capital sobre la creación de empresas. Loría, Ramírez y Salas (2014) incorporan un índice de flexibilidad laboral en México, sobre el crecimiento del desempleo, con una muestra trimestral entre 1997Q3 y 2014Q1.

Como vemos, la literatura no es ajena al hecho de incorporar otras variables que midan las relaciones subyacentes entre el desempleo y el producto, y es esa la vía que buscamos explorar. 


\section{METODOLOGÍA}

\section{Ecuaciones estimadas}

En este artículo se desarrollan únicamente los dos primeros modelos de Okun, con un total de ocho estimaciones (E), cuatro por cada modelo. El primer modelo es el de primeras diferencias, y asume una relación entre el diferencial de las tasas de desempleo como variable dependiente sobre la base de la variación porcentual del PBI real (DLPBI). En las estimaciones 1 y 2, las variaciones en las tasas de desempleo (DTD) son únicamente referidas al desempleo abierto; y en las estimaciones [3] y [4] las tasas de desempleo son sumadas a las tasas de subempleo (TDA $)$.

$$
\begin{array}{ll}
D T D_{t}=\alpha_{0}+\alpha_{1} D L P B I_{t}+\alpha_{i} \sum_{i=1}^{n} X_{i t}+\varepsilon_{t^{\prime}} & \varepsilon_{t} \sim N I D\left(0, \sigma^{2}\right) \text { (Estimación 1 y 2) } \\
D T D_{t}=\beta_{0}+\beta_{1} D L P B I_{t}+\beta_{i} \sum_{i=1}^{n} X_{i t}+\tau_{t^{\prime}} & \tau_{t} \sim N I D\left(0, \sigma^{2}\right) \text { (Estimación } 3 \text { y } 4 \text { ) }
\end{array}
$$

Las variables adicionales incluidas $\left(x_{i t}\right)$ fueron la tasa inflación, y variables ficticias que dan cuenta de valores atípicos puntuales y persistentes.

El segundo modelo es el de enfoque de brechas, para lo cual se calculó, a través del filtro de Hodrick y Prescott, la tasa porcentual de desempleo natural y el PBI potencial ${ }^{9}\left({ }{ } I_{t}^{*}\right)$, y con este último la brecha de producto $\left(G_{A P}{ }_{t}\right)$. Este modelo asume una relación entre la brecha de la tasa de desempleo como variable dependiente sobre la base de la brecha del PBI real, respecto de su nivel potencial. La diferencia de las estimaciones se refiere, igual que antes, a que en las estimaciones 5 y 6 la brecha de la tasa de desempleo, que viene a ser la del desempleo cíclico (TDC $C_{t}$, se refiere únicamente al desempleo abierto; y en las estimaciones 7 y 8 , la tasa de desempleo cíclico se calcula a partir de la ampliación hecha para sumarle la tasa de subempleo ( $\left.T D A C_{t}\right)$, que como se explicó, intenta agregar una medida del bidireccional efecto de desplazamiento (de los adecuadamente empleados al subempleo, y viceversa):

$$
\begin{gathered}
T D C_{t}=\theta_{0}+\theta_{1} G A P_{t}+\theta_{i} \sum_{i=1}^{n} X_{i t}+\gamma_{t^{\prime}} \quad \gamma_{t} \sim N I D\left(0, \sigma^{2}\right)(\text { Estimación } 5 \text { y } 6) \\
T D A C_{t}=\delta_{0}+\delta_{1} G A P_{t}+\delta_{i} \sum_{i=1}^{n} X_{i t}+\varphi_{t^{\prime}} \quad \varphi_{t} \sim N I D\left(0, \sigma^{2}\right)(\text { Estimación } 7 y 8) \\
\text { Siendo: GAPt }=\frac{P B I_{t}-P B I_{t}^{*}}{P B I_{t}^{*}}, T D C_{t}=T D_{t}-T D_{t}^{*} \text { y } T D A C_{t}=T D A_{t}-T D A_{t}^{*}
\end{gathered}
$$

9 Bajo este método de estimación del PBI potencial, nos alejamos de la postura keynesiana de Okun, de capacidad productiva a la tasa de pleno empleo, y más bien seguimos la línea monetarista dominante de tasa natural de desempleo, entendida como desviaciones ante choques externos, pudiendo tomar valores negativos y positivos, lo cual es importante para su interpretación. 
En este modelo, las variables adicionales incluidas $\left(x_{i t}\right)$ fueron la tasa inflación, la variación porcentual de la productividad laboral y variables facticias que dan cuenta de valores atípicos puntuales y persistentes. La variable en asterisco (PIB $\left.{ }_{t}^{*}\right)$ denota el producto tendencial y las de $T D_{t}{ }^{*} \mathrm{y} T D A_{t}{ }_{t}$ se refieren a las tasas naturales de desempleo y desempleo ampliado (desempleo abierto más subempleo).

\section{Análisis de los datos}

Las variables relacionadas con el mercado laboral y demás variables explicativas fueron obtenidas principalmente del Instituto Nacional de Estadística e Informática (INEI), Ministerio de Trabajo y Promoción de Empleo (MTPE), Banco Central de Reserva del Perú (BCRP), Organización Internacional del Trabajo (OIT), Banco Mundial (BM), Fondo Monetario Internacional (FMI), Comisión Económica para América Latina (CEPAL) y de bases de datos halladas en trabajos de investigación previos, y de publicaciones de series estadísticas.

En la tabla 1 se presentan las variables de interés en el presente trabajo, ${ }^{10}$ las fuentes de donde han sido obtenidas y la unidad en que están expresadas. La frecuencia de las variables es anual y abarca desde 1970 hasta 2018.

Tabla 1.

Resumen de variables originales seleccionadas (1970-2018) 1/

\begin{tabular}{|c|c|c|c|}
\hline Variables & Fuente & Nomenclatura & Unidades \\
\hline Tasa de desempleo & MTPE & td & Tasa porcentual sobre PEA \\
\hline Tasa de subempleo & MTPE & ts & Tasa porcentual sobre PEA \\
\hline Tasa de desempleo ampliado & $\begin{array}{c}\text { Elaboración a partir de } \\
(\mathrm{D}+\mathrm{S}) / \text { PEA }\end{array}$ & tda & Tasa porcentual sobre PEA \\
\hline PBI real $(2007=100)$ & BCRP & PBI & En millones de soles \\
\hline Inflación & BCRP & inf & Tasa porcentual \\
\hline Productividad laboral $(2007=100)$ & PBI real / PEA empleada & prod & En soles \\
\hline
\end{tabular}

Fuente: elaboración del autor.

Algunos valores de las variables ligadas al mercado de trabajo han sido construidos a partir de diversos documentos y estadísticas parciales, publicados física o virtualmente por diversas entidades a lo largo de las casi cinco décadas que abarcan las series, y analizadas con la rigurosidad que exige una investigación científica,

10 En el anexo 1, al final del artículo, pueden verse las series de las variables básicas incluidas en los modelos. 
teniendo cuidado de contrastar la congruencia y darles consistencia a las diversas medidas y metodologías de recolección de los datos a través del tiempo.

En la figura 4 se presenta la evolución gráfica de las variables de desempleo y desempleo ampliado (desempleo más subempleo), junto con las variables explicativas, tasa de variación porcentual del producto (DLPBI), brecha relativa del producto (GAP), que son variables construidas a partir de la serie PBI real, inflación y tasa de variación porcentual de la productividad laboral, que se utilizarán en los modelos.

Figura 4. Gráfica de las series relevantes en el análisis
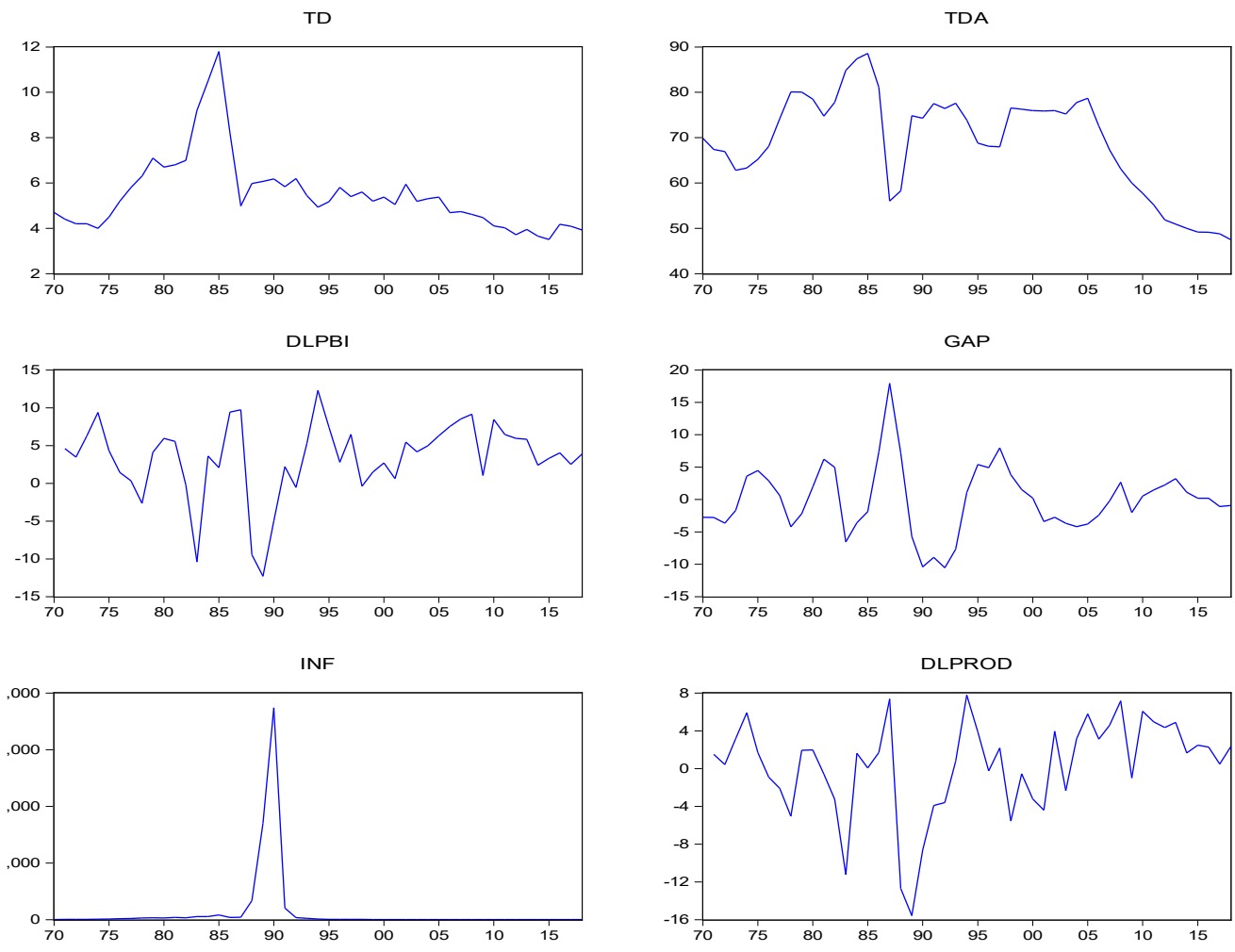

Fuente: elaboración del autor. 
De igual forma, se presenta el resumen estadístico de las variables en la tabla 2 , donde se observa que, salvo la variable tasa de desempleo ampliado, las demás no se ajustan a una distribución normal.

Tabla 2.

\section{Estadísticas descriptivas de variables relevantes}

\begin{tabular}{|c|c|c|c|c|c|c|}
\hline & TD & TDA & DLPBI & GAP & INF & DLPROD \\
\hline Mean & 5,5 & 69,0 & 3,3 & 0,0 & 275,1 & 0,3 \\
\hline Median & 5,2 & 73,2 & 4,1 & 0,2 & 9,0 & 1,6 \\
\hline Maximum & 11,8 & 88,6 & 12,3 & 17,9 & 7481,7 & 7,8 \\
\hline Minimum & 3,5 & 47,5 & $-12,3$ & $-10,6$ & 0,2 & $-15,6$ \\
\hline Std. Dev. & 1,7 & 11,1 & 5,0 & 5,2 & 1172,4 & 5,1 \\
\hline Skewness & 1,8 & $-0,5$ & $-1,3$ & 0,6 & 5,4 & $-1,2$ \\
\hline Kurtosis & 7,0 & 2,2 & 5,0 & 4,7 & 32,1 & 4,4 \\
\hline Jarque-Bera & 58,7 & 3,0 & 21,2 & 7,9 & 1928,3 & 14,7 \\
\hline Probability & 0,00 & 0,22 & 0,00 & 0,02 & 0,00 & 0,00 \\
\hline Sum & 265 & 3310 & 160 & $-0,6$ & 13.205 & 14,3 \\
\hline Sum Sq. Dev. & 132 & 5814 & 1.170 & $1,27 \mathrm{E}+03$ & 64.602 .660 & $1,20 \mathrm{E}+03$ \\
\hline Observations & 48 & 48 & 48 & 48 & 48 & 48 \\
\hline
\end{tabular}

Fuente: elaboración del autor.

Previo a la estimación de los modelos de Okun, en la tabla 3 se presenta la matriz de correlaciones entre las variables relevantes. Se comprueba en una primera instancia que los signos de cada variable dependiente sobre el PBI cumplen con lo anticipado por la teoría económica; por consiguiente, se espera que los signos de los coeficientes de los modelos sean los adecuados.

Tabla 3.

Matriz de correlaciones de variables relevantes

\begin{tabular}{|c|c|c|c|c|c|c|}
\hline & TD & TDA & DLPBI & GAP & INF & DLPROD \\
\hline TD & 1 & 0,764 & $-0,310$ & $-0,143$ & 0,106 & $-0,350$ \\
\hline TDA & 0,764 & 1 & $-0,207$ & $-0,348$ & 0,108 & $-0,332$ \\
\hline DLPBI & $-0,310$ & $-0,207$ & 1 & 0,372 & $-0,456$ & 0,946 \\
\hline GAP & $-0,143$ & $-0,348$ & 0,372 & 1 & $-0,333$ & 0,349 \\
\hline INF & 0,106 & 0,108 & $-0,456$ & $-0,333$ & 1 & $-0,474$ \\
\hline PROD & $-0,350$ & $-0,332$ & 0,946 & 0,349 & $-0,474$ & 1 \\
\hline
\end{tabular}

Fuente: elaboración del autor. 
Las variables de desempleo abierto y subempleo fueron sometidas al análisis de la prueba de raíz unitaria de Dickey Fuller Aumentada (ADF), asumiendo constante y tendencia en el análisis, cuyos resultados se presentan en la tabla 4.

Tabla 4

Pruebas de Raíz Unitaria (ADF) de variables de desempleo y subempleo

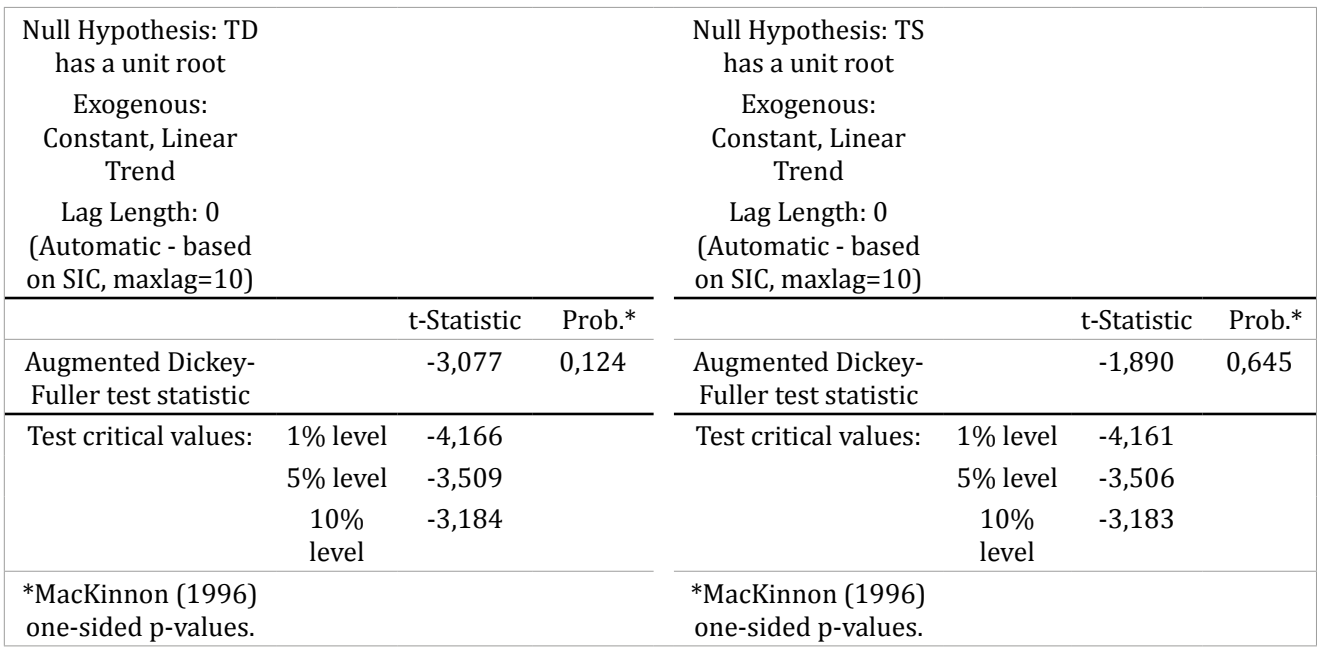

Fuente: elaboración del autor.

Observamos que no se rechaza la hipótesis nula de que dichas variables tienen raíz unitaria; por tanto, son no estacionarias, y se confirma la hipótesis económica de que existe el fenómeno de histéresis en el desempleo y en el subempleo.

\section{RESULTADOS}

\section{Modelo de primeras diferencias}

Del modelo de primeras diferencias surgen cuatro estimaciones (E1, E2, E3 y E4), que se presentan en la tabla $5 .{ }^{11}$ En todas se cumple con el supuesto de normalidad

11 Como variables explicativas adicionales en los modelos propuestos, únicamente se incluyeron las variables inflación y productividad laboral, aunque también se probó la relevancia de algunas otras variables en los modelos 2, 4, 6 y 8, como las horas-hombre perdidas en huelgas en el sector privado, la formación bruta de capital, el índice de gasto no financiero del Gobierno General, y dos variables dicotómicas, la primera de migración y la segunda de flexibilidad laboral, las cuales finalmente no resultaron significativas. 
de los residuos, buena especificación lineal, ${ }^{12}$ no autocorrelación serial y residuos homocedásticos, aplicándose en todos los casos el método de MCO. Además, todas las estimaciones presentaron ajustes muestrales razonablemente altos $\left(R^{2}\right)$ y ajustes globales significativos (F). Todos los coeficientes del producto tienen los signos esperados y resultan significativos al 99\%.

En las estimaciones 1 y 2 , los resultados muestran que el coeficiente de Okun para el Perú en su versión de primeras diferencias es de -0,058 y -0,072, respectivamente, 0 dicho de otra forma, que un aumento marginal de 1\% del PBI (ceteris paribus) genera una reducción de 5,8 y 7,2 puntos básicos en la variación de la tasa de desempleo abierto.

Por otra parte, si igualamos a cero el diferencial de la tasa de desempleo ( $\mathrm{dtd}=0$ ), podemos despejar el valor requerido mínimo de variación porcentual del producto, asumiendo que todas las demás variables no cambian de su último valor del 2018 para que se cumpla dicha igualdad. De esta forma, encontramos que para que la tasa de desempleo abierto se reduzca, el PBI real (ceteris paribus) debería crecer en la estimación 1, al menos 5,34\% y en la estimación 2 al menos un 5,39\%, ambas cifras similares y mayores a las alcanzadas por Okun y otros autores para países desarrollados.

Tabla 5.

Resultados del modelo de primeras diferencias

\begin{tabular}{|c|c|c|c|c|}
\hline Estimaciones: & (E1) & $(\mathrm{E} 2)$ & (E3) & (E4) \\
\hline Variable dependiente: & $\begin{array}{l}\text { Variación de tasa } \\
\text { de desempleo (dtd) }\end{array}$ & $\begin{array}{l}\text { Variación de tasa } \\
\text { de desempleo (dtd) }\end{array}$ & $\begin{array}{c}\text { Variación de tasa de } \\
\text { desempleo y subempleo } \\
\text { (dtda) }\end{array}$ & $\begin{array}{c}\text { Variación de tasa de } \\
\text { desempleo y subempleo } \\
\text { (dtda) }\end{array}$ \\
\hline \multicolumn{5}{|l|}{ Variables principales: } \\
\hline - Constante & 0,309 & 0,390 & 1,436 & 2,071 \\
\hline - Var\%PBI (dlpbi) & $-0,058 * * *$ & $-0,072 * * *$ & $-0,490 * * *$ & $-0,576 * * *$ \\
\hline \multicolumn{5}{|l|}{ Otras variables: } \\
\hline - Var\%PBI*dummy89 & & & $-0,742 * * *$ & $-0,876 * * *$ \\
\hline - Desempleo + Subempleo rezagado $\left(\mathrm{dtda}_{\mathrm{t}-1}\right)$ & & & & $0,169 * *$ \\
\hline - Inflación (Inf) & & $0,000 *$ & & $-0,001 * *$ \\
\hline - dummy86 & $-3,364 * * *$ & $-3,298 * * *$ & & \\
\hline - dummy87 & $-2,963 * * *$ & $-2,892 * * *$ & $-21,800 * * *$ & $-20,249 * * *$ \\
\hline Adjusted R-squared & 0,678 & 0,693 & 0,780 & 0,815 \\
\hline Sum squared resid & 11,959 & 11,132 & 289,044 & 231,006 \\
\hline Log likelihood & $-34,756$ & $-33,037$ & $-111,198$ & $-104,109$ \\
\hline Prob(F-statistic) & 0,000 & 0,000 & 0,000 & 0,000 \\
\hline DW & 1,680 & 1,907 & 1,827 & 2,211 \\
\hline
\end{tabular}

Fuente: elaboración del autor.

12 Las pruebas de normalidad de los residuos y de buena especificación de las estimaciones finales se presentan en los Anexos del 2 al 5, al final del artículo. 
En las estimaciones 3 y 4, los resultados muestran que el coeficiente de Okun para el Perú, en nuestra adaptación funcional de desempleo sumado al subempleo, en su versión de primeras diferencias, es de -0,490 y -0,576, respectivamente; es decir, que un aumento marginal de $1 \%$ del PBI real (ceteris paribus) genera una reducción de 49 y 58 puntos básicos en la variación de la tasa de desempleo ampliado con subempleo. En vista del rezago del desempleo ampliado dentro de la estimación 4, el efecto total (de corto y largo plazo) en el desempleo, sobre la base del producto, es de $-0,693$. $^{13}$

Operando de igual manera a como hicimos antes, igualamos a cero el diferencial de la tasa de desempleo ampliado ( $\mathrm{dtda}=0$ ), asumiendo que todas las demás variables no cambian de su último valor del 2018 para que se cumpla dicha igualdad. Entonces, para que el desempleo ampliado se reduzca, el PBI real (ceteris paribus) debería crecer al menos 2,93\% en la estimación 3 y al menos 3,22\% en la estimación 4; cifras menores a las obtenidas con las estimaciones 1 y 2 , lo cual vendría explicado por el efecto desplazamiento que se genera en el subempleo.

De hecho, cuando la economía crece, aumenta el total de personas empleadas, tanto en empleos adecuados como en el subempleo; pero en proporción a la PEA, la tasa de subempleo, al igual que la tasa de desempleo, se reducen por el fenómeno que hemos denominado de desplazamiento (traslado del subempleo hacia el empleo adecuado).

Como se observa en la tabla 6, los intervalos de confianza de los coeficientes de la tasa de variación porcentual del producto (DLPBI) para el nivel de confianza del $99 \%$ en las primeras cuatro estimaciones se yuxtaponen, y las pruebas aplicadas demuestran que el efecto de dicha variable es el mismo dentro de cada grupo de estimaciones del modelo.

Finalmente, aunque el valor del coeficiente de la inflación en la estimación 4 es bastante pequeño (-0.001), al igual que su valor en la estimación 2 , su signo es contrario a lo esperado, lo cual podría ser explicado por los periodos de inflación persistente que tuvo el Perú durante casi dos décadas y que redujo la creación de puestos de trabajo adecuados y fomentó el aumento de empleos precarios (vía reducción de horas de trabajo y reemplazo de trabajadores con menores salarios).

13 El efecto total sería igual al coeficiente de dlpbi / (1-coefiente de dtda $\left.{ }_{\mathrm{t}-1}\right)=-0.576 /(1-0.169)$ 
Tabla 6.

Intervalos de confianza del coeficiente de Var\%PBI

\begin{tabular}{|c|c|c|c|c|c|c|c|c|}
\hline \multirow{2}{*}{ Estimación } & \multirow{2}{*}{ Variable } & \multirow{2}{*}{ Coefficient } & \multicolumn{2}{|c|}{$90 \% \mathrm{CI}$} & \multicolumn{2}{|c|}{$95 \% \mathrm{CI}$} & \multicolumn{2}{|c|}{$99 \%$ CI } \\
\hline & & & Low & High & Low & High & Low & High \\
\hline Estimación 1 & DLPBI & $-0,058$ & $-0,084$ & $-0,031$ & $-0,090$ & $-0,026$ & $-0,100$ & $-0,015$ \\
\hline Estimación 2 & DLPBI & $-0,072$ & $-0,102$ & $-0,043$ & $-0,107$ & $-0,037$ & $-0,119$ & $-0,025$ \\
\hline \multirow{2}{*}{ Estimación } & \multirow{2}{*}{ Variable } & \multirow{2}{*}{ Coefficient } & \multicolumn{2}{|c|}{$90 \% \mathrm{CI}$} & \multicolumn{2}{|c|}{$95 \% \mathrm{CI}$} & \multicolumn{2}{|c|}{$99 \% \mathrm{CI}$} \\
\hline & & & Low & High & Low & High & Low & High \\
\hline Estimación 3 & DLPBI & $-0,490$ & $-0,635$ & $-0,345$ & $-0,664$ & $-0,316$ & $-0,722$ & $-0,257$ \\
\hline Estimación 4 & DLPBI & $-0,576$ & $-0,720$ & $-0,431$ & $-0,749$ & $-0,402$ & $-0,808$ & $-0,344$ \\
\hline
\end{tabular}

Fuente: elaboración del autor.

\section{Modelo de enfoque de brechas}

De las especificaciones del segundo modelo usando el enfoque de brechas, surgen también cuatro estimaciones (E5, E6, E7 y E8), las que se presentan en la tabla 7. En todas se observa una buena especificación lineal y se cumple con los supuestos de normalidad de los residuos. En la estimación 5, para corregir la autocorrelación, se aplicó un modelo lineal de correlación serial en el término de error de primer orden AR(1), ${ }^{14}$ a través del procedimiento recursivo de Cochrane Orcutt; en la estimación 7 se aplicó el método robusto de estimación de la varianza de Newey West, que supera los problemas de autocorrelación y homocedasticidad al mismo tiempo; y en la estimación 8 se aplicó el método robusto de varianzas y errores estándar consistentes con heterocedasticidad de White que supera dicho problema.

Además, todas las estimaciones presentaron ajustes muestrales razonablemente altos $\left(\mathrm{R}^{2}\right)$ y ajustes poblaciones elevados (F). Todos los signos de los coeficientes de brecha de producción son los esperados, y significativos al 99\%.

En las estimaciones 5 y 6, los resultados muestran que el coeficiente de Okun en su versión de brechas es de -0,054 y-0,042, respectivamente; dicho de otra forma, que un crecimiento marginal de $1 \%$ del GAP (ceteris paribus) provoca una reducción del desempleo ampliado respecto de su tasa natural de 5,4 puntos básicos en la

14 Es una metodología que describe una clase particular de proceso en el que los errores en un momento $t$ dado son estimados por los errores rezagados de forma recursiva. El valor del coeficiente representa el coeficiente de autocorrelación (rho), y no requiere ninguna interpretación. 
estimación 5 y de 4,2 puntos básicos en la estimación 6, valores que son inferiores a los encontrados por Garavito (2003) para dicho modelo. ${ }^{15}$

Por otra parte, que la brecha de desempleo asociada a un gap de cero (gap=0), manteniendo constante el resto de las variables ancladas al último escenario de 2018, es de $-0,05 \%$ para la estimación 5 y de - $0,07 \%$ para la estimación 6 , lo cual confirma lo que establecen diversos autores: que en el estado estacionario se cierra la brecha de desempleo, es decir, que el desempleo se aproxima a la tasa de desempleo natural o en términos dinámicos a la NAIRU. ${ }^{16}$

Tabla 7.

Resultados del modelo de enfoque de brechas

\begin{tabular}{|c|c|c|c|c|}
\hline Estimaciones: & (E5) $1 /$ & (E6) & $(\mathrm{E} 7) 2 /$ & $(\mathrm{E} 8) 3 /$ \\
\hline Variable dependiente: & $\begin{array}{c}\text { tasa de desempleo } \\
\text { cíclico } \\
\text { (tdc) }\end{array}$ & $\begin{array}{c}\text { tasa de desempleo } \\
\text { cíclico } \\
\text { (tdc) }\end{array}$ & $\begin{array}{l}\text { tasa de desempleo y } \\
\text { subempleo cíclico } \\
\text { (tdac) }\end{array}$ & $\begin{array}{c}\text { tasa de desempleo y } \\
\text { subempleo cíclico } \\
\text { (tdac) }\end{array}$ \\
\hline Variables principales: & & & & \\
\hline - Constante & $-0,046$ & $-0,067$ & 0,217 & 0,314 \\
\hline - Output gap (gap) & $-0,054 * * *$ & $-0,042 * * *$ & $-0,423 * * *$ & $-0,307 * * *$ \\
\hline \multicolumn{5}{|l|}{ Otras variables: } \\
\hline - Output gap*dummy87 & $-0,085 * * *$ & $-0,088 * *$ & & \\
\hline - Desempleo + Subempleo rezagado $\left(\mathrm{tdac}_{\mathrm{t}-1}\right)$ & & & $0,502 * * *$ & $0,627 * * *$ \\
\hline - Inflación (Inf) & & $0,000 * *$ & & \\
\hline - Var\% de la productividad laboral real & & & & $-0,225 * *$ \\
\hline - dummy84 & $1,649 * * *$ & $2,492 * * *$ & & \\
\hline - dummy85 & $3,127 * * *$ & $3,943 * * *$ & & \\
\hline - dummy87 & & & $-14,325 * * *$ & $-15,449 * * *$ \\
\hline$-\operatorname{AR}(1)$ & $0,576 * * *$ & & & \\
\hline Adjusted R-squared & 0,778 & 0,760 & 0,741 & 0,851 \\
\hline Sum squared resid & 7,589 & 8,202 & 237,999 & 173,139 \\
\hline Log likelihood & $-24,033$ & $-25,737$ & $-114,233$ & $-98,899$ \\
\hline Prob(F-statistic) & 0,000 & 0,000 & 0,000 & 0,000 \\
\hline DW & 1,973 & 1,590 & 1,502 & 2,017 \\
\hline
\end{tabular}

Fuente: elaboración del autor.

En las estimaciones 7 y 8, los resultados muestran que el coeficiente de Okun para el Perú en nuestra adaptación funcional de desempleo ampliado, en su versión de brechas, es de $-0,423$ y $-0,307$, respectivamente; dicho de otra forma, que

15 El análisis pionero realizado por dicha autora abarca del año 1970 al 2000. Aunque no presenta un análisis de quiebre estructural, señala que los datos indican que la relación entre el empleo y el producto es consistente en el tiempo, mientras que antes de 1990 las dos series, si bien crecientes, no se mueven juntas (p 19).

16 Para una mayor referencia de la NAIRU (tasa de desempleo no aceleradora de la inflación) y su relación de esta con el producto potencial, puede revisarse: Cuadrado et al. (2010, p. 208). 
un crecimiento marginal de 1\% del GAP (ceteris paribus) provoca una reducción del desempleo ampliado respecto de su tasa natural de 42,3 puntos básicos en la estimación 7 y 30,7 puntos básicos en la estimación 8. Considerando que ambos modelos incorporan el rezago de la variable dependiente, el efecto total de la brecha del producto sobre la brecha del desempleo sería de $-0,849^{17}$ y $-0,823^{18}$ para las estimaciones 7 y 8 , respectivamente.

Por otra parte, que la brecha de desempleo asociada a un gap de cero (gap=0), manteniendo constante el resto de las variables ancladas al último escenario de 2018, es de 1,32\% y 1,17\% para la estimación 7 y 8, respectivamente. En este caso, el resultado es positivo y superior a los obtenidos con el desempleo abierto, lo que implica que el desempleo ampliado resulta mayor que su tendencia de largo plazo.

Como se observa en la tabla 8, los intervalos de confianza de los coeficientes del GAP para el nivel de confianza del $99 \%$ en las cuatro estimaciones agrupadas sobre el desempleo abierto (E5 y E6) y desempleo ampliado (E7 y E8) se yuxtaponen, y las pruebas aplicadas demuestran que el efecto de dicha variable es el mismo dentro de cada grupo de estimaciones del modelo.

Tabla 8.

Intervalos de confianza del coeficiente de brecha de producto

\begin{tabular}{|l|c|c|c|c|c|c|c|c|c|}
\hline \multirow{2}{*}{ Estimación } & \multirow{2}{*}{ Variable } & \multirow{2}{*}{ Coefficient } & \multicolumn{2}{|c|}{$90 \%$ CI } & \multicolumn{2}{c|}{$95 \%$ CI } & \multicolumn{2}{c|}{$99 \%$ CI } \\
\cline { 4 - 10 } & & Low & High & Low & High & Low & High \\
\hline Estimación 5 & GAP & $-0,054$ & $-0,085$ & $-0,024$ & $-0,091$ & $-0,017$ & $-0,103$ & $-0,005$ \\
\hline Estimación 6 & GAP & $-0,042$ & $-0,068$ & $-0,016$ & $-0,073$ & $-0,011$ & $-0,083$ & 0,000 \\
\hline & & & & & & & & \\
\hline
\end{tabular}

Fuente: elaboración del autor.

Finalmente, el coeficiente de la productividad laboral real en la estimación 8 tiene un impacto negativo sobre la brecha de desempleo de $-0,225$, que va con lo esperado en el marco conceptual.

17 El efecto total en la estimación 7 sería igual al coeficiente del gap / (1-coefiente de tdact- 1$)=-0.423 /(1-0.502)$

18 El efecto total en la estimación 8 sería igual al coeficiente del gap / (1-coefiente de tdact-1) $=-0.307 /(1-0.627)$ 


\section{CONCLUSIONES}

A pesar de haber transcurrido más de medio siglo desde el documento inicial sobre la ley de Okun, continúa siendo de gran interés para la política económica, por cuanto esta sirve como una métrica rápida para la toma de decisiones y de guía a los agentes económicos.

En este artículo se han aplicado los dos primeros modelos de Okun y se ha demostrado econométricamente que, en el modelo de primeras diferencias usando como variable dependiente la variación de la tasa del desempleo abierto, para que la tasa de desempleo abierto se reduzca, el PBI debe crecer en promedio al menos un 5,37\%, mayor valor al encontrado por Okun y otros autores para economías desarrolladas.

Cuando se analiza con la variación de la tasa de desempleo ampliado (desempleo más subempleo) como variable dependiente, para que la tasa de desempleo más subempleo se reduzca, el PBI debe crecer en promedio al menos un 3,07\%, menor valor al encontrado únicamente para el desempleo abierto como variable dependiente, lo cual demuestra que el subempleo (como proxi del empleo de mala calidad) sirve como válvula de escape y contención social, evidenciando además lo que hemos llamado el efecto desplazamiento.

Respecto del segundo modelo, considerando como variable dependiente la brecha de la tasa de desempleo abierto, cuando la brecha de producto se cierra $(\mathrm{GAP}=0)$, aun así, la brecha en la tasa de desempleo en promedio se reduce en $-0,06 \%$, algo explicado por otros factores.

Cuando se analiza la brecha sobre la tasa de desempleo ampliado como variable dependiente (desempleo más subempleo), y la brecha del producto se cierra $(\mathrm{GAP}=0)$, la brecha en la tasa de desempleo ampliado en promedio se reduce $1,24 \%$.

Estos resultados reafirman parcialmente las estimaciones hechas por Garavito en el 2003 para el caso peruano y por otros autores para países en vías de desarrollo, que se alejan de las estimaciones hechas para países desarrollados, y revelan que, para salir de la trampa de los países de ingresos medios, se requiere ampliar más rápidamente la capacidad productiva del país, por lo que no basta con políticas de demanda.

Se demostró, además, que la tasa de desempleo y la tasa de desempleo ampliada son variables no estacionarias, y por tanto, que estadísticamente los rezagos 
de la variable influyen sobre su valor presente, es decir, que presentan el fenómeno de histéresis.

Esta investigación representa un punto de partida para el análisis de la estructura del mercado de trabajo y su relación con el crecimiento económico; sin embargo, deja abiertas dos ampliaciones metodológicas que pueden ser exploradas. La primera, que considere la asimetría del coeficiente de Okun respecto del crecimiento económico y que podría ser hecha a través de una estimación no lineal; la segunda, aquella que pueda estimar el producto potencial y desempleo natural a partir de una metodología de estimación multivariante o de función de producción.

\section{AGRADECIMIENTOS}

Deseo expresar mi agradecimiento a las autoridades de la Universidad de Lima, en especial a la directora María Teresa Quiroz, a cargo del Instituto de Investigación Científica (IDIC), por la oportunidad y el apoyo que han brindado a este trabajo, y la confianza ofrecida. Un trabajo de investigación siempre es fruto de ideas y esfuerzos previos que se comparten con otras personas; en tal sentido, agradezco también a todos mis colegas de la carrera de Economía que me brindaron sus valiosos comentarios. Asimismo, agradezco al Comité Editorial de la Revista Finanzas y Política Económica, y a los evaluadores anónimos, que con sus valiosos comentarios me ayudaron a mejorar diversos aspectos del contenido y bien podrían ser considerados coautores de este documento.

Finalmente, agradecer a mi asistente de investigación María Rita Huarancca por su dedicación, que fue fundamental en la recolección y construcción de la información estadística.

Esta investigación fue posible gracias al apoyo económico del Instituto de Investigación Científica de la Universidad de Lima (IDIC), como parte de su convocatoria al concurso de Proyectos de Investigación 2019.

\section{CONFLICTOS DE INTERÉS}

El autor declara que no existe ningún conflicto de interés relacionado con el artículo y que todas aquellas relaciones de carácter financiero establecidas con cualquier organización o entidad, cuyos productos o servicios están directamente relacionados con el contenidodel manuscrito, se encuentran expresamente declaradas. 


\section{REFERENCIAS}

1. Almonte, L. y Carbajal Suárez Y. (2011). Crecimiento económico y desempleo en el Estado de México: una relación estructural. Urbe. Revista Brasileira de Gestão Urbana (Brazilian Journal of Urban Management), 3(1), 77-88.

2. Anderton, R., Aranki, T., Bonthuis, B. y Jarvis, V. (2014). Disaggregating Okun's law: decomposing the impact of the expenditure components of GDP on euro area unemployment (Working Paper Series 1747). European Central Bank. https://doi.org/10.2866/36377

3. Ball, L., Leigh, D. y Loungani, P. (2012). Okun's Law: Fit at 50? 13th Jacques Polak Annual Research Conference. FMI. Noviembre 8-9, 2012. https://doi. org/10.5089/9781475574265.001

4. Bande, R. y Martín Román, Á. L. (2018). Regional differences in the Okun's relationship: New evidence for Spain (1980-2015). Investigaciones Regionales - Journal of Regional Research, (41), 137-165. https://ideas.repec.org/a/ris/invreg/0375.html

5. Bardales, J. (2011). Informalidad y subempleo: evidencia microeconométrica para el caso peruano. Horizonte Económico, (1). Instituto de Investigación de la Facultad de Ciencias Económicas de la Universidad Nacional del Callao. http://fce.unac.edu.pe/ images/investigacion/unidad-investigacion/revista/he-n1/a11.pdf

6. Belapatiño, V., Grippa F. y Perea, H. (2017, 9 de enero). Perú: Informalidad laboral y algunas propuestas para reducirla. Observatorio Económico Perú. BBVA. https://www.bbvaresearch.com/wp-content/uploads/2017/01/Observatorio-informalidad-laboral1.pdf

7. Blanchard, O., Amighini, A. y Giavazzi, F. (2012). Macroeconomía. Madrid: Pearson Educación.

8. Cazes, S., Verick, S. y Al Hussami, F. (2013). Why did unemployment respond so differently to the global financial crisis across countries? Insights from Okun's Law. IZA Journal of Labor Policy, (2), 10. https://doi.org/10.1186/2193-9004-2-10

9. Céspedes, N. (2003). Factores cíclicos y estructurales en la evolución de la tasa de desempleo. Revista Estudios Económicos, (9), 199-221.

10. Céspedes, N. y Ramírez, R. (2016). Estimación de la productividad total de los factores en el Perú: Enfoques primal y dual. Productividad en el Perú: Medición, determinantes e implicancias (1. ${ }^{\text {a }}$ ed.). Lima: Universidad del Pacífico. https://doi. org/10.21678/978-9972-57-356-9-1

11. Chacaltana, J. (2000). Un análisis dinámico del desempleo en el Perú [Mimeo]. Lima: Fondo de Investigaciones del Programa MECOVI, INEI. https://www.grade.org.pe/ publicaciones/139-un-analisis-dinamico-del-desempleo-en-el-peru

12. Chávez Muñoz, N. M. (2010). Inflación y crecimiento económico: determinantes del desempleo en Colombia. Revista Finanzas y Política Económica, 2(1), 29-52. https:// revfinypolecon.ucatolica.edu.co/article/view/543

13. Cuadrado, J. R, Mancha, T., Villena, J. E., Casares, J., González, M., Marín, J. M. ${ }^{\mathrm{a}}$ y Peinado, M. (2010). Política Económica: Elaboración, objetivos e instrumentos (4.a ed.). Madrid: McGraw-Hill/ Interamericana de España. 
Una revisión para el Perú de la relación entre el desempleo, el subempleo y la producción

14. Daly, M y Hobijn, B. (2010). Okun's Law and the Unemployment Surprise of 2009. FRBSF Economic Letter. 2010-07 March 8, 2010.

15. Fortin, B. y Bernier, A. (1988). The welfare cost of unemployment in Quebec: Harberger's triangle meets Okun's gap. Canadian Journal of Economics, 21 (1), 161-181. https://doi.org/10.2307/135217

16. Garavito, C. (2003). La ley de Okun en el Perú. Revista Economía, (26), 51-52). http:// revistas.pucp.edu.pe/index.php/economia/article/view/731

17. Gordon, R. J. (1984). Unemployment and Potential Output in the 1980s. Brookings Papers on Economic Activity, (2). https://doi.org/10.2307/2534438

18. Hamada, K. y Kurosaka, Y. (1984). The relationship between production and unemployment in Japan: Okun's law in comparative perspective. European Economic Review, 25(1), 71-94. https://doi.org/10.1016/0014-2921(84)90073-4

19. Holloway, T. (1989). An Updated Look at Okun's Law. Social Science Quarterly, 70 (2), 497-504. www.jstor.org/stable/42862585

20. Instituto Nacional de Estadística e Informática (INEI). (2017). Producción y Empleo Informal en el Perú, Cuenta Satélite de la Economía Informal 2007-2016. https:// www.inei.gob.pe/media/MenuRecursivo/publicaciones_digitales/Est/Lib1589/libro.pdf

21. Instituto Nacional de Estadística e Informática (INEI). (2018). Perú: Indicadores de Empleo e Ingreso por Departamento, 2007-2017. https:/www.inei.gob.pe/media/ MenuRecursivo/publicaciones_digitales/Est/Lib1537/index.html

22. Izyumov, A. y Vahaly, J. (2002). The Unemployment-Output Tradeoff in Transition Economies: Does Okun's Law Apply? Economics of Planning, 35(4), 317-331. https:// doi.org/10.1023/A:1024441219635

23. Jiménez, K. y Ochoa, W. S. (2017). ley de Okun. Análisis de la relación entre crecimiento y desempleo para 12 países de América Latina. Revista Publicando, 4(13). 1-15.

24. Knoester, A. (1986). Okun's law revisited. Weltwirtschaftliches Archiv, (122), 657-666. https://doi.org/10.1007/BF02707853

25. Knotek II, E. S. (2007). How useful is Okun's law? Economic Review-Federal Reserve Bank of Kansas City, 92(4), 73. https://www.kansascityfed.org/publicat/econrev/ pdf/4q07knotek.pdf

26. Loría Díaz de Guzmán, E., Ramírez, E. y Salas, E. (2015). La ley de Okun y la flexibilidad laboral en México: un análisis de cointegración, 1997Q3-2014Q1. Revista Internacional de Contaduría y Administración, (60), 631-650. https://doi.org/10.1016/j. cya.2015.05.012

27. Loría, E. y Ramos, M. G. (2007). La ley de Okun: una relectura para México, 1970-2004. Estudios Económicos, 22 (1), 19-55. https://www.redalyc.org/articulo.oa?id=59722102

28. Organización Internacional del Trabajo (OIT). (2018). Panorama Laboral de América Latina y el Caribe 2018. Lima: OIT / Oficina Regional para América Latina y el Caribe, 2018. https://www.oitcinterfor.org/node/7458

29. Okun, A. M. (1962). Potential GNP: Its Measurement and Significance. Proceedings of the Business and Economic Statistics Section, Washington, D.C., pp. 98-104. 
30. Paéz-Cortés, J. N., (2013). Una revisión de la ley de Okun para Latinoamérica (tesis doctoral). Cali: Universidad del Valle.

31. Prachowny, M. F. (1993). Okun's Law: Theoretical Foundations and Revised Estimates. The Review of Economics and Statistics, (75), 331-336. https://doi.org/10.2307/2109440

32. Román, A. M. y Porras, S. (2012). La ley de Okun en España ipor qué existen diferencias regionales? November 2012. Conference: XXXVIII Reunión de Estudios Regionales. https://doi.org/10.13140/2.1.1674.3204

33. Távara, Gonzales de Olarte y Del Pozo (2014). Heterogeneidad estructural y articulación productiva en el Perú: Evolución y estrategias. Hacia un desarrollo inclusivo: El caso del Perú. Chacaltana, J. y Infante, R. Editores. CEPAL/OIT. Pp. 39-79. https://repositorio. cepal.org/bitstream/handle/11362/40739/LCL3779_es.pdf?sequence $=1$ \&isAllowed $=y$

34. Unevska Andonova, D. y Petrovska, M. (2019). Disaggregating Okun's Law: A CaseStudy for Macedonia. Journal of Central Banking Theory and Practice, (1), 183-207. https://doi.org/10.2478/jcbtp-2019-0009

35. Valadkhani, A. (2015). Okun's Law in Australia. Economic Record, 91 (295), 509-522. https://doi.org/10.1111/1475-4932.12221 
Una revisión para el Perú de la relación entre el desempleo, el subempleo y la producción

Anexo 1: Series empleadas en el presente artículo 1/

\begin{tabular}{|c|c|c|c|c|c|}
\hline Año & TD & TDA & PBI & INF & PROD \\
\hline 1970 & 4,7 & 69,8 & 116.849 & 5,0 & 29.422 \\
\hline 1971 & 4,4 & 67,4 & 122.213 & 6,8 & 29.862 \\
\hline 1972 & 4,2 & 66,9 & 126.463 & 7,2 & 29.990 \\
\hline 1973 & 4,2 & 62,8 & 134.401 & 9,5 & 30.940 \\
\hline 1974 & 4,0 & 63,3 & 147.017 & 16,9 & 32.773 \\
\hline 1975 & 4,5 & 65,2 & 153.340 & 23,6 & 33.330 \\
\hline 1976 & 5,2 & 68,1 & 155.559 & 33,5 & 33.030 \\
\hline 1977 & 5,8 & 74,2 & 156.102 & 38,0 & 32.336 \\
\hline 1978 & 6,3 & 80,1 & 151.977 & 57,9 & 30.699 \\
\hline 1979 & 7,1 & 80,0 & 158.194 & 67,7 & 31.291 \\
\hline 1980 & 6,7 & 78,5 & 167.596 & 59,2 & 31.907 \\
\hline 1981 & 6,8 & 74,8 & 176.901 & 75,4 & 31.721 \\
\hline 1982 & 7,0 & 77,8 & 176.507 & 64,5 & 30.688 \\
\hline 1983 & 9,2 & 84,8 & 158.136 & 111,1 & 27.236 \\
\hline 1984 & 10,5 & 87,4 & 163.842 & 110,2 & 27.676 \\
\hline 1985 & 11,8 & 88,6 & 167.219 & 163,4 & 27.695 \\
\hline 1986 & 8,2 & 81,1 & 182.981 & 77,9 & 28.164 \\
\hline 1987 & 5,0 & 56,0 & 200.778 & 85,8 & 30.243 \\
\hline 1988 & 6,0 & 58,2 & 181.822 & 667,0 & 26.404 \\
\hline 1989 & 6,1 & 74,8 & 159.436 & $3.398,3$ & 22.290 \\
\hline 1990 & 6,2 & 74,3 & 151.492 & $7.481,7$ & 20.363 \\
\hline 1991 & 5,8 & 77,5 & 154.854 & 409,5 & 19.566 \\
\hline 1992 & 6,2 & 76,4 & 154.017 & 73,5 & 18.863 \\
\hline 1993 & 5,4 & 77,6 & 162.093 & 48,6 & 19.009 \\
\hline 1994 & 4,9 & 73,9 & 182.044 & 23,7 & 20.489 \\
\hline 1995 & 5,2 & 68,8 & 195.536 & 11,1 & 21.284 \\
\hline 1996 & 5,8 & 68,1 & 201.009 & 11,5 & 21.229 \\
\hline 1997 & 5,4 & 68,0 & 214.028 & 8,5 & 21.690 \\
\hline 1998 & 5,6 & 76,5 & 213.190 & 7,3 & 20.484 \\
\hline 1999 & 5,2 & 76,3 & 216.377 & 3,5 & 20.372 \\
\hline 2000 & 5,4 & 76,0 & 222.207 & 3,8 & 19.714 \\
\hline 2001 & 5,1 & 75,9 & 223.580 & 2,0 & 18.848 \\
\hline 2002 & 5,9 & 75,9 & 235.773 & 0,2 & 19.592 \\
\hline 2003 & 5,2 & 75,2 & 245.593 & 2,3 & 19.131 \\
\hline 2004 & 5,3 & 77,8 & 257.770 & 3,7 & 19.738 \\
\hline 2005 & 5,4 & 78,7 & 273.971 & 1,6 & 20.881 \\
\hline 2006 & 4,7 & 72,6 & 294.598 & 2,0 & 21.530 \\
\hline 2007 & 4,7 & 67,3 & 319.693 & 1,8 & 22.518 \\
\hline 2008 & 4,6 & 63,1 & 348.923 & 5,8 & 24.132 \\
\hline 2009 & 4,5 & 60,0 & 352.584 & 2,9 & 23.892 \\
\hline 2010 & 4,1 & 57,7 & 382.380 & 1,5 & 25.340 \\
\hline 2011 & 4,0 & 55,2 & 407.052 & 3,4 & 26.592 \\
\hline 2012 & 3,7 & 51,9 & 431.273 & 3,7 & 27.750 \\
\hline 2013 & 4,0 & 50,9 & 456.449 & 2,8 & 29.104 \\
\hline 2014 & 3,7 & 50,0 & 467.433 & 3,2 & 29.587 \\
\hline 2015 & 3,5 & 49,2 & 482.890 & 3,5 & 30.320 \\
\hline 2016 & 4,2 & 49,1 & 502.341 & 3,6 & 31.006 \\
\hline 2017 & 4,1 & 48,8 & 514.927 & 2,8 & 31.156 \\
\hline 2018 & 3,9 & 47,5 & 534.867 & 1,3 & 31.882 \\
\hline
\end{tabular}

1/ Tasas porcentuales de las variables del mercado de empleo sobre la Población Económicamente Activa (PEA).

Fuente: elaboración del autor. 


\section{Anexo 2: Pruebas de normalidad de los residuos de la ecuación (modelos de primeras diferencias)}
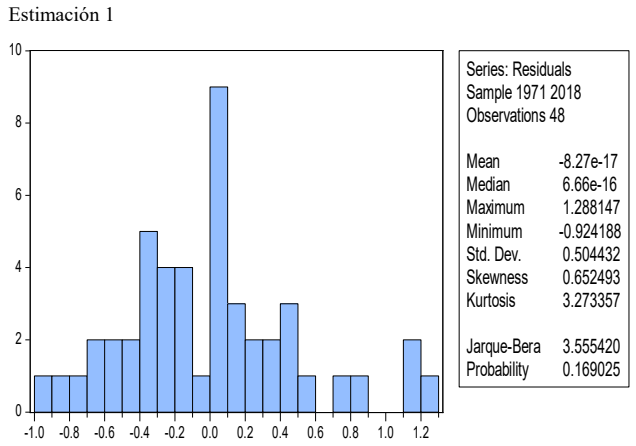

Estimación 2

Estimación 3
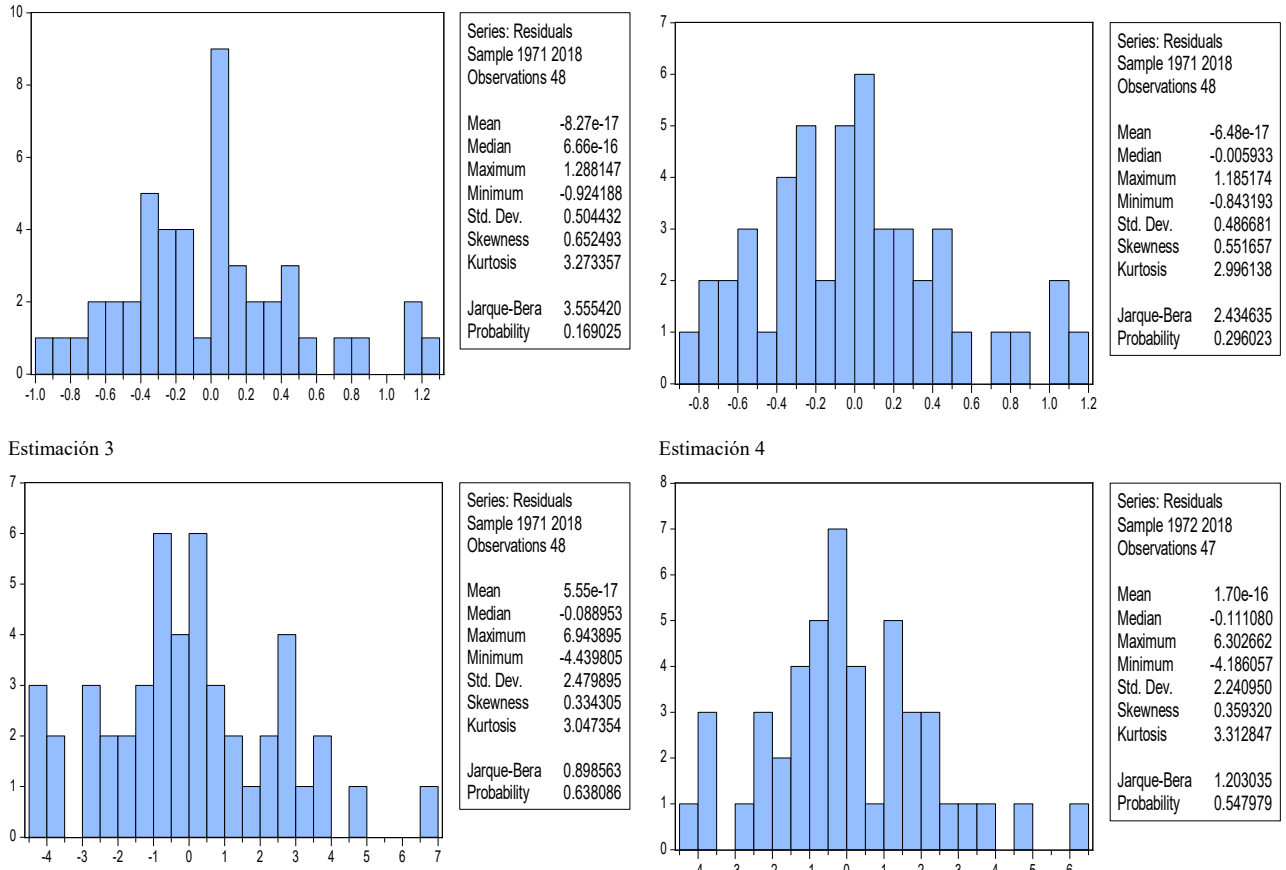

Estimación 4

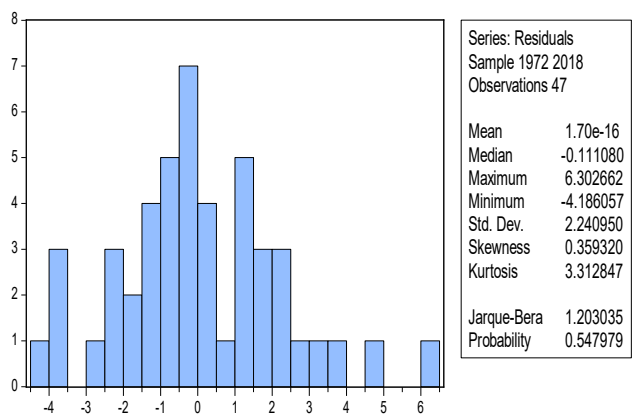

Fuente: elaboración del autor. 


\section{Anexo 3: Pruebas de normalidad de los residuos de la ecuación (modelos de brechas)}
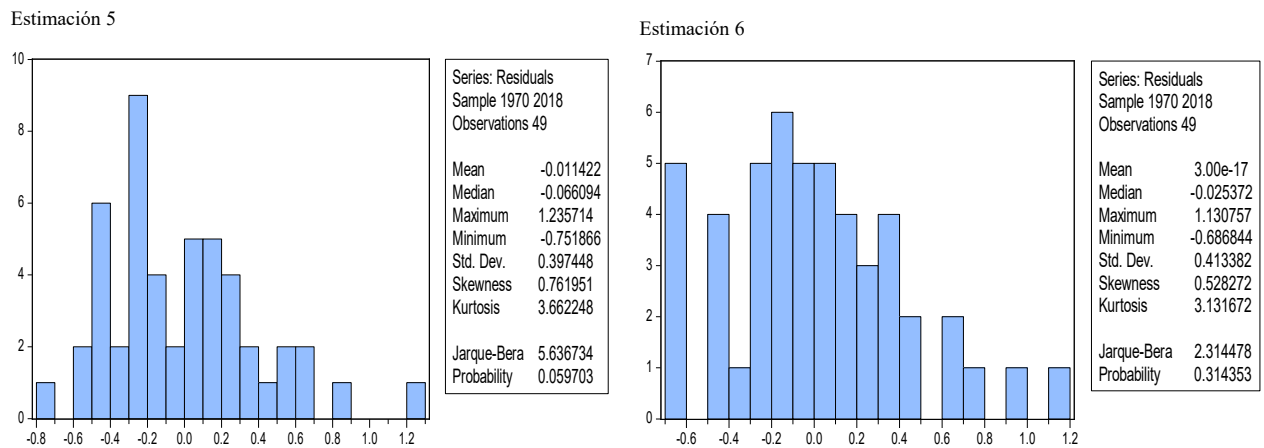

Estimación 7

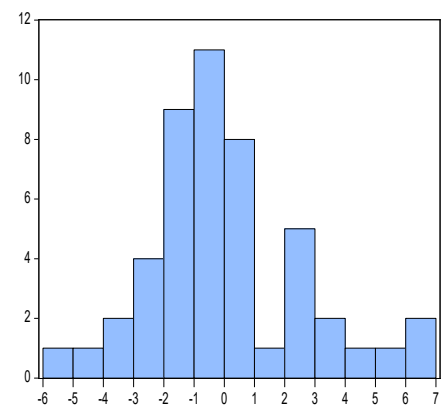

\begin{tabular}{|ll|}
\hline \multicolumn{2}{|l|}{ Series: Residuals } \\
Sample 19712018 \\
Observations 48 \\
Mean & $-2.17 \mathrm{e}-16$ \\
Median & -0.247857 \\
Maximum & 6.584266 \\
Minimum & -5.954774 \\
Std. Dev. & 2.641725 \\
Skewness & 0.544920 \\
Kurtosis & 3.410365 \\
& \\
Jarque-Bera & 2.712305 \\
Probability & 0.257650 \\
\hline
\end{tabular}

Estimación 8

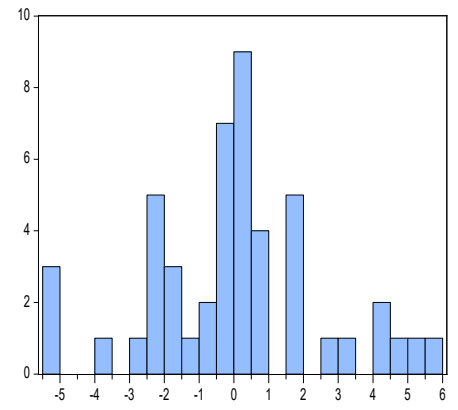

Series: Residuals Sample 19712018

Observations 48

Mean $\quad-9.25 \mathrm{e}-17$

Median $\quad 0.032746$

Maximum 5.545349

Ninimum $\quad-5.404964$

Std. Dev. $\quad 2.500810$

Skewness $\quad 0.088141$

Kurtosis $\quad 3.200281$

Jarque-Bera $\quad 0.142376$ Probability $\quad 0.931287$

Fuente: elaboración del autor. 


\section{Anexo 4: Pruebas de adecuada especificación (modelos de primeras diferencias)}

Estimación 1

Ramsey RESET Test

Equation: EQ1A

Specification: DTD C DLPBI D86 D87

Omitted Variables: Powers of fitted values from 2 to 6

\begin{tabular}{lccc}
\hline \hline & Value & df & Probability \\
\cline { 2 - 4 } F-statistic & $1.62018 \ldots$ & $(5,39)$ & 0.1775 \\
Likelihood ratio & $9.05910 \ldots$ & 5 & 0.1067 \\
\hline \hline F-test summary: & \multicolumn{3}{l}{} \\
& Sum of S... & df & Mean Squares \\
Test SSR & $2.05687 \ldots$ & 5 & 0.411375 \\
Restricted SSR & $11.9592 \ldots$ & 44 & 0.271800 \\
Unrestricted SSR & $9.90232 \ldots$ & 39 & 0.253906 \\
\hline \hline LR test summary: & \multicolumn{3}{|l}{} \\
& Value & df & \\
Restricted LogL & $-34.7562 \ldots$ & 44 & \\
Unrestricted LogL & $-30.2267 \ldots$ & 39 & \\
\hline \hline
\end{tabular}

Estimación 3

\begin{tabular}{|c|c|c|c|}
\hline $\begin{array}{l}\text { Ramsey RESET T } \\
\text { Equation: EQ3A } \\
\text { Specification: DTD } \\
\text { Omitted Variables }\end{array}$ & $\begin{array}{l}\text { DLPBI DLPB } \\
\text { ers of fitted } v\end{array}$ & $\begin{array}{l}889 \text { D87 } \\
\text { ues from }\end{array}$ & \\
\hline & Value & df & Probability \\
\hline F-statistic & $1.85663 \ldots$ & $(3,41)$ & 0.1521 \\
\hline Likelihood ratio & $6.11435 \ldots$ & 3 & 0.1062 \\
\hline F-test summary: & & & \\
\hline & Sum of S... & $\mathrm{df}$ & Mean Squares \\
\hline Test SSR & $34.5705 \ldots$ & 3 & 11.52352 \\
\hline Restricted SSR & $289.044 \ldots$ & 44 & 6.569190 \\
\hline Unrestricted SSR & $254.473 \ldots$ & 41 & 6.206678 \\
\hline LR test summary: & & & \\
\hline & Value & $\mathrm{df}$ & \\
\hline Restricted LogL & $-111.198 \ldots$ & 44 & \\
\hline Unrestricted LogL & $-108.141 \ldots$ & 41 & \\
\hline
\end{tabular}

Estimación 2

Ramsey RESET Test

Equation: $\mathrm{EQ} 2 \mathrm{~A}$

Specification: DTD C DLPBI D86 D87 INF

Omitted Variables: Powers of fitted values from 2 to 4

\begin{tabular}{|c|c|c|c|}
\hline & Value & df & Probability \\
\hline F-statistic & $2.45889 \ldots$ & $(3,40)$ & 0.0767 \\
\hline Likelihood ratio & $8.12403 \ldots$ & 3 & 0.0435 \\
\hline \multicolumn{4}{|l|}{ F-test summary: } \\
\hline & Sum of S... & df & Mean Squares \\
\hline Test SSR & $1.73334 \ldots$ & 3 & 0.577780 \\
\hline Restricted SSR & $11.1323 \ldots$ & 43 & 0.258892 \\
\hline Unrestricted SSR & $9.39902 \ldots$ & 40 & 0.234976 \\
\hline \multicolumn{4}{|l|}{ LR test summary: } \\
\hline & Value & df & \\
\hline Restricted LogL & $-33.0367 \ldots$ & 43 & \\
\hline Unrestricted LogL & $-28.9747 \ldots$ & 40 & \\
\hline
\end{tabular}

Estimación 4

Ramsey RESET Test

Equation: EQ4A

Specification: DTDA C DLPBI DLPBI*D89 DTDA(-1) D87 INF Omitted Variables: Powers of fitted values from 2 to 3

\begin{tabular}{lccc}
\hline \hline & Value & df & Probability \\
\cline { 2 - 4 } F-statistic & $0.52749 \ldots$ & $(2,39)$ & 0.5942 \\
Likelihood ratio & $1.25450 \ldots$ & 2 & 0.5341 \\
\hline \hline
\end{tabular}

\begin{tabular}{lccc}
\hline \hline F-test summary: & & \multicolumn{2}{c}{} \\
& Sum of S... & df & Mean Squares \\
\cline { 2 - 4 } Test SSR & $6.08436 \ldots$ & 2 & 3.042180 \\
Restricted SSR & $231.005 \ldots$ & 41 & 5.634281 \\
Unrestricted SSR & $224.921 \ldots$ & 39 & 5.767209 \\
\hline \hline \multirow{2}{*}{ LR test summary: } & & \multicolumn{2}{c}{} \\
& Value & df & \\
Restricted LogL & $-104.109 \ldots$ & 41 & \\
Unrestricted LogL & $-103.481 \ldots$ & 39 & \\
\hline \hline
\end{tabular}

Fuente: elaboración del autor. 
Una revisión para el Perú de la relación entre el desempleo, el subempleo y la producción

\section{Anexo 5: Pruebas de adecuada especificación (modelos de brechas)}

Estimación 5

\begin{tabular}{|c|c|c|c|}
\hline $\begin{array}{l}\text { Ramsey RESET Te } \\
\text { Equation: EQ5A } \\
\text { Specification: TDC } \\
\text { Omitted Variables: }\end{array}$ & $\begin{array}{l}\text { AP GAP*D87 } \\
\text { ares of fitted }\end{array}$ & $\begin{array}{l}84 \text { D85 } \\
\text { lues }\end{array}$ & $R(1)$ \\
\hline & Value & $\mathrm{df}$ & Probability \\
\hline t-statistic & $1.95289 \ldots$ & 42 & 0.0575 \\
\hline F-statistic & $3.81377 \ldots$ & $(1,42)$ & 0.0575 \\
\hline Likelihood ratio & $4.32775 \ldots$ & 1 & 0.0375 \\
\hline \multicolumn{4}{|l|}{ F-test summary: } \\
\hline & Sum of S... & df & Mean Squares \\
\hline Test SSR & $0.63172 \ldots$ & 1 & 0.631723 \\
\hline Restricted SSR & $7.58869 .$. & 43 & 0.176481 \\
\hline Unrestricted SSR & $6.95697 \ldots$ & 42 & 0.165642 \\
\hline \multicolumn{4}{|l|}{ LR test summary: } \\
\hline & Value & df & \\
\hline Restricted LogL & $-24.0333 \ldots$ & 43 & \\
\hline Unrestricted LogL & $-21.8694 \ldots$ & 42 & \\
\hline
\end{tabular}

Estimación 7

Ramsey RESET Test

Equation: EQ7A

Specification: TDAC C GAP TDAC(-1) D87

Omitted Variables: Powers of fitted values from 2 to 4

\begin{tabular}{|c|c|c|c|}
\hline & Value & df & Probability \\
\hline F-statistic & $2.63992 \ldots$ & $(3,41)$ & 0.0622 \\
\hline Likelihood ratio & $8.47724 \ldots$ & 3 & 0.0371 \\
\hline \multicolumn{4}{|l|}{ F-test summary: } \\
\hline & Sum of S... & df & Mean Squares \\
\hline Test SSR & $53.1007 \ldots$ & 3 & 17.70026 \\
\hline Restricted SSR & $327.999 \ldots$ & 44 & 7.454532 \\
\hline Unrestricted SSR & $274.898 \ldots$ & 41 & 6.704845 \\
\hline \multicolumn{4}{|l|}{ LR test summary: } \\
\hline & Value & $\mathrm{df}$ & \\
\hline Restricted LogL & $-114.232 \ldots$ & 44 & \\
\hline Unrestricted LogL & $-109.993 \ldots$ & 41 & \\
\hline
\end{tabular}

Estimación 6

\begin{tabular}{|c|c|c|c|}
\hline $\begin{array}{l}\text { Ramsey RESET T } \\
\text { Equation: EQ6B } \\
\text { Specification: TDC } \\
\text { Omitted Variables: }\end{array}$ & AP GAP*D87 & $\begin{array}{l}84 \text { D85 } \\
\text { ues from }\end{array}$ & $\begin{array}{l}\text { NF } \\
\text { to } 3\end{array}$ \\
\hline & Value & df & Probability \\
\hline F-statistic & $1.85955 \ldots$ & $(2,41)$ & 0.1686 \\
\hline Likelihood ratio & $4.25462 \ldots$ & 2 & 0.1192 \\
\hline F-test summary: & & & \\
\hline & Sum of S... & df & Mean Squares \\
\hline Test SSR & $0.68216 \ldots$ & 2 & 0.341083 \\
\hline Restricted SSR & $8.20245 \ldots$ & 43 & 0.190755 \\
\hline Unrestricted SSR & $7.52028 \ldots$ & 41 & 0.183422 \\
\hline LR test summary: & & & \\
\hline & Value & df & \\
\hline Restricted LogL & $-25.7370 \ldots$ & 43 & \\
\hline Unrestricted LogL & $-23.6096 \ldots$ & 41 & \\
\hline
\end{tabular}

Estimación 8

Ramsey RESET Test

Equation: EQ8B

Specification: TDAC C GAP D87 TDAC(-1) DLPROD

Omitted Variables: Powers of fitted values from 2 to 3

\begin{tabular}{|c|c|c|c|}
\hline & Value & df & Probability \\
\hline F-statistic & $3.00176 \ldots$ & $(2,41)$ & 0.0607 \\
\hline Likelihood ratio & $6.55923 \ldots$ & 2 & 0.0376 \\
\hline \multicolumn{4}{|l|}{ F-test summary: } \\
\hline & Sum of S... & $\mathrm{df}$ & Mean Squares \\
\hline Test SSR & $37.5436 \ldots$ & 2 & 18.77180 \\
\hline Restricted SSR & $293.940 \ldots$ & 43 & 6.835824 \\
\hline Unrestricted SSR & $256.396 \ldots$ & 41 & 6.253581 \\
\hline \multicolumn{4}{|l|}{ LR test summary: } \\
\hline & Value & $\mathrm{df}$ & \\
\hline Restricted LogL & $-111.601 \ldots$ & 43 & \\
\hline Unrestricted LogL & $-108.321 \ldots$ & 41 & \\
\hline
\end{tabular}

Fuente: elaboración del autor. 
